\title{
EFECTO DE LOMBRICES DE TIERRA (Pontoscolex corethrurus) SOBRE EL CRECIMIENTO DE CULTIVOS ANUALES Y CARACTERISTICAS FISICAS Y QUIMICAS EN SUELOS DE YURIMAGUAS
}

Beto Pashanasi *

Patrick Lavelle ${ }^{* *}$

Julio A legre**

\section{RESUMEN}

Este ensayo fue conducido en la E.E. "San Ramón" de Y urimaguas, en un suelo Típico paleudult Fino Siliceo hypertérmico, Perú, con el objeto de evaluar el efecto de Pontoscolex corethrurus sobre el crecimiento y producción de 6 ciclos de cultivos anuales y las propiedades del suel 0.

El ensayo tuvo un diseño factorial de $3 \times 2$ con 3 tratamientos de manejo de residuos: $C$ : control; $C R$ : residuos de cosecha $\left(2.5 \mathrm{~T} \mathrm{hal}^{-1)}\right.$, y CRB: residuos de cosecha + abono verde de leguminosa (2.5 T ha ${ }^{-1}$, de cada uno); y 2 subtratamientos de lombrices (sin lombrices o con un inóculo de $360 \mathrm{~kg} \mathrm{ha}^{-1}$ de Pontoscolex corethrurus de peso fresco).

La producción fue significativamente afectada por la introducción de lombrices y el tipo y cantidad de insumos orgánicos. Los más al tos rendimientos en promedio se obtuvieron en los tratamientos con residuos de cultivo + abono verde de leguminosa e inoculación de lombrices de $1.62 \mathrm{~T}^{\text {ha-1 }}$ en 6 cosechas sucesivas (112\% más que el control sin lombriz, y sin residuo de cosecha) (0.77 $\mathrm{T}^{\mathrm{n}} \mathrm{a}^{-1}$ ).

Efectos significativos de las lombrices sobre la producción de las plantas fue observada desde el primer cultivo (maíz) con un incremento promedio de $37 \%$. En el segundo cultivo (arroz), el incremento en promedio fue de $88 \%$; 10 contrario en la tercera cosecha (caupí), no mostró efecto significativo y un decrecimiento de $-5 \%$. En la cuarta y sexta cosecha (arroz) mostré efectos significativos sobre el promedio con incremento de 50 y $52 \%$ respectivamente. En la quinta cosecha (arroz), mostró un efecto negativo con un decrecimiento en promedio de $-43 \%$ quizás debido a un accidental período seco.

Después del primer cultivo, el contenido de $\mathrm{C}$ del suelo decreció constantemente en todos los tratamientos con lombriz hasta el sexto cultivo.

IN IA. Estación Experimental "San Ramón”, Y urimaguas, Perú.

** Laboratoire d'E cologie des Sols Tropicaux. ORSTOM. Paris, Francia

*** International Centre for Research in Agroforesty (ICRAF). Yurimaguas, Perú. 
El contenido de $\mathrm{N}$ fue mayor en los tratamientos con lombrices en las tres primeras cosechas, siendo la tendencia invertida en la quinta cosecha aunque no hubo diferencia significativa; manteniéndose la tendencia hasta la sexta cosecha.

El incremento de la densidad aparente fue altamente significativa en los tratamientos con lombriz. La tasa de infiltración decrece significativamente con el tiempo en todos los tratamientos, pero con un ligero incremento en la sexta cosecha.

La dinámica de los nutrientes del suelo con lombrices fue similar a los tratamientos sin lombriz. Hubo un incremento inicial durante el primer cultivo debido a la adición de nutrientes en las cenizas.

\section{INTRODUCCION}

A nivel mundial los bosques tropicales cubren 2970 millones de hectáreas. Durante el presente siglo su área ha disminuido significativamente; se estima que el año 2000 serán talados e intervenidas por el hombre unos 225 millones de hectáreas más. La destrucción de los bosques tropicales tiene grandes repercusiones para la humanidad debido al aumento de $\mathrm{CO} 2$ en la atmósfera y pérdida de la biodiversidad dando el efecto de invernadero.

En la A mazonía donde los suelos bien drenados son de baja fertilidad, estas pérdidas se deben evitar (Cochrame y Sánchez, 1982).

La causa principal de la tala está en la expansión de la "Frontera agrícola" para ganar nuevas tierras de cultivo a través del sistema de agricultura migratoria. Este sistema es el más difundido en el trópico húmedo para el cultivo de plantas alimenticias anuales. Consiste en la eliminación de la cobertura boscosa mediante la tumba y quema. Las cenizas y el material parcialmente quemado producen un elevado aporte de nutrientes al suelo, donde hay aumento en el $\mathrm{pH}, \mathrm{P}, \mathrm{Ca}, \mathrm{Mg}$ y K intercambiables, así como una disminución del $\mathrm{Al}$ intercambiable. Así el suelo desnudo queda totalmente expuesto a procesos de erosión, que son mayores cuando las pendientes son muy pronunciadas, las Iluvias son intensas y la capacidad de almacenamiento y permeabilidad del suelo son bajos. También hay un rápido deterioro de las propiedades físicas del suelo, mediante la destrucción de los agregados los cuales dependen de la textura, el contenido de materia orgánica, la actividad y descomposición de raíces, la actividad de los organismos del suelo, los cationes adsorbidos por las partículas del suelo y el uso de la tierra (Estribi y Chavarría, 1984).

La presión demográfica actual impone períodos de barbecho y descanso más cortos (3 a 4 años), lo que significa una disminución de la recuperación al 
potencial de fertilidad del suelo debido a un período más corto de reciclaje, por tal motivo es necesario mejorar los barbechos en su etapa de regeneración natural. Por esto es necesario adoptar tecnologías que permitan mantener 0 mejorar la fertilidad del suelo a largo plazo. La manipulación de los procesos biológicos del suelo es una de las vías más prometedoras para lograr esta meta. Entre dichos procesos, se puede aprovechar la actividad de las lombrices de tierra endógenas 0 anécicas, que viven en el suelo al contrario de las epígeas que viven en la acumulación de materia orgánica y no penetran en el suelo. La fauna del suelo puede también jugar un papel importante en este proceso de agregación (Blanchart et al, 1990). Las lombrices epigeas se pueden utilizar para el “compost de materia orgánica (lombricultura), pero no pueden vivir en el suelo.

Las lombrices endógenas contribuyen al mantenimiento de la fertilidad, pues construyen y mantienen una estructura en base a macroagregados resistentes, y también liberan nutrientes a partir de residuos vegetales y de la materia orgánica del suelo, y por último, protegen físicamente el humus dentro de sus turrículos compactos.

Las lombrices generalmente están ausentes en los cultivos anuales y algunas plantaciones perennes (por ejemplo plantación de té) porque las especies nativas no están adaptadas a condiciones de suelo cultivado y las especies foráneas adaptadas a condiciones de suelo cultivado y las especies foráneas adaptadas no han sido capaces de colonizar (Figura 1) (Critchley et al 1979, Lavelle y Pashanasi 1989, Dangerfield 1990, Barois et al 1991, Lavelle y Fragoso 1992). Al mismo tiempo es posible que la colonización sea extremadamente lenta (casi $10 \mathrm{~m}$ por año en promedio) y detenidos por algunos obstáculos pequeños como ríos o bosques. El rápido declive de la fertilidad en el sistema de agricultura tradicional puede ser atribuido en parte a la escasez de regulación por los macroinvertebrados especialmente lombrices.

El objetivo principal de este estudio fue: evaluar el efecto de las lombrices Pontoscolex corethrurus sobre el crecimiento y producción de cultivos anuales; y evaluar su efecto sobre el mantenimiento y/o mejoramiento de la fertilidad de los suelos. 


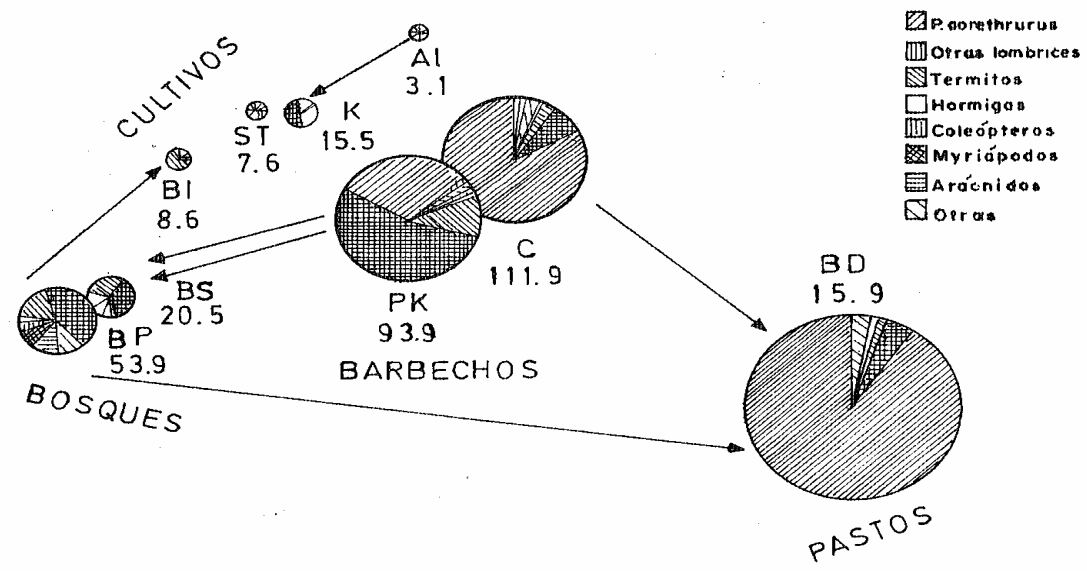

Figura 1. Distribución de biomasa entre las principales unidades taxonómicas en diferentes tipos de uso del suelo: BP Bosque primario; $\mathrm{BS}=$ Bosque secundario; $\mathrm{BD}=$ BrachiariaDesmodium; $C=$ centrosema pubescens, $P K=$ Pijuayo-Kudzu; $\mathrm{BI}=$ Bajos insumos; $\mathrm{Al}=\mathrm{Altos}$ insumos; $\mathrm{ST}=$ Sistema tradicional.

\section{MATERIALES Y METODOS}

Este estudio se llevó a cabo entre los años 1990 y 1992, en la Estación Experimental "San Ramón" de Y urimaguas ubicado a 50 56` latitud Sur, 76ำ longitud Oeste a una elevación de 184 msnm.

El clima es húmedo tropical, con una temperatura promedio de $26^{\circ} \mathrm{C}$ y una precipitación pluvial anual de $2100 \mathrm{~mm}$ (Figura 2). Hay una época seca corta entre junio y agosto donde los totales mensuales de lluvia están por debajo de 100 $\mathrm{mm}$. El suelo es un típico Paleudult con una textura franco arcillo arenoso (23\% de arcilla, $55 \%$ de arena y $22 \%$ de limo). 
Tiene de $\mathrm{pH} 4.0$, un porcentaje de saturación de aluminio del $94 \%$, y bajo contenido de nutrientes especialmente en $\mathrm{P}$ aprovechable $(9 \mathrm{ppm}) ; \mathrm{K}$ $(0.07 \mathrm{cmol} / 100)$ y $\mathrm{Mg}(0.10 \mathrm{cmol} / / 00)$.

A ntes de la instalación de las parcelas, se hizo un muestreo cuantitativo de la macrofauna del suelo utilizando el método de muestreo propuesto por el Programa "Tropical Soil Biology and Fertility" (TSBF). Se clasificó la macrofauna de acuerdo a su categoría ecológica y se midió la biomasa de cada grupo de organismos.

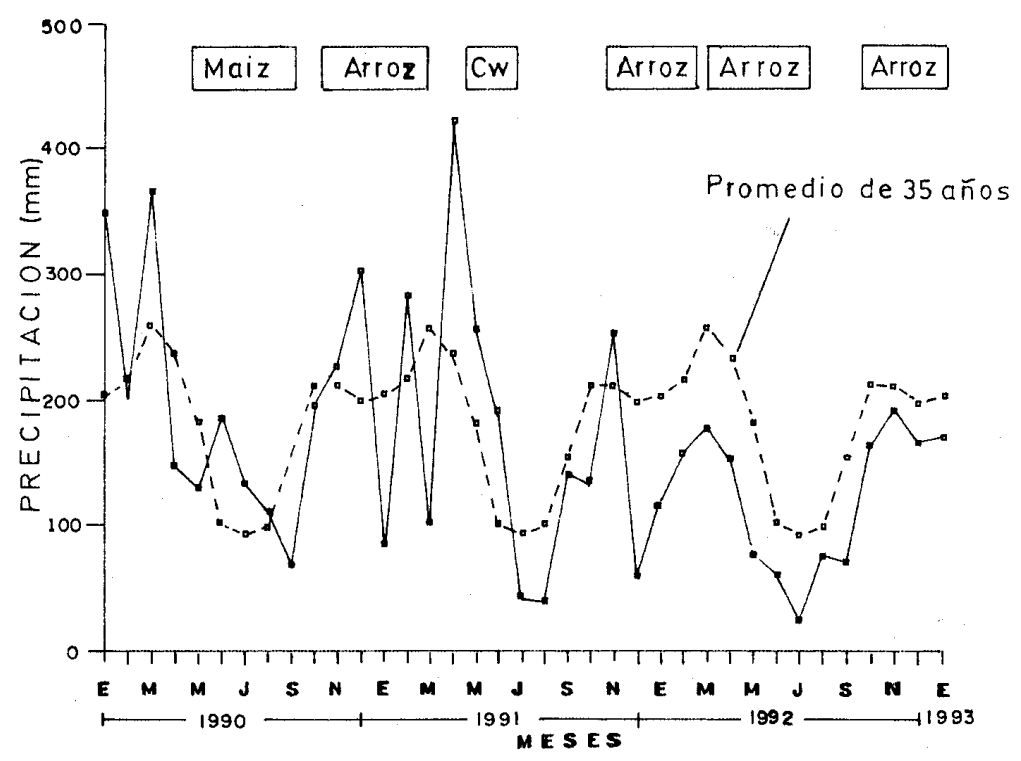

Figura 2. Precipitación $(\mathrm{mm})$ mensual durante el período del experimento en la estación Experimental "San Ramón" de Y urimaguas, L oreto-Perú. 
Se encontró una densidad de 1719 individuos $\mathrm{m}^{-2}$ y una biomasa de $30.8 \mathrm{~g} \mathrm{~m}^{-2}$ de peso fresco, similar a la biomasa encontrada en el año de 1986 (Lavelle y Pashanasi 1989) en un bosque secundario de 20 años aproximadamente (Cuadro 1)

\section{Cuadro 1}

Promedio de abundancia y biomasa de los macroinvertebrados del bosque secundario de la parcela experimental.

\begin{tabular}{lllll}
\hline & $\begin{array}{l}\text { A bundancia } \\
\text { Ind. } \mathrm{m}^{2}\end{array}$ & $\begin{array}{l}\text { Biomasa } \\
\text { g.m }\end{array}$ & $\begin{array}{l}\text { A bundancia } \\
\text { Ind. } \mathrm{m}^{2}\end{array}$ & $\begin{array}{l}\text { Biomasa } \\
\text { g.m }{ }^{2}\end{array}$ \\
\hline Lombrices & 137.0 & 18.4 & 85.0 & 11.3 \\
S.E & $(8.2)$ & $(876.3)$ & $(39.0)$ & $(4.0)$ \\
Termites & 766.0 & 1.6 & 3570.0 & 6.9 \\
S.E & $(84.3)$ & $(183.8)$ & $(1842.6)$ & $(2.7)$ \\
Hormigas & 475.0 & 1.3 & 290.0 & 0.7 \\
S.E & $(15.1)$ & $(115.6)$ & $(94.5)$ & $(0.5)$ \\
Coleópteros & 66.0 & 2.9 & 29.0 & 0.6 \\
S.E & $(3.2)$ & $(284.8)$ & $(10.9)$ & $(0.3)$ \\
A rácnidos & 87.0 & 1.0 & 45.0 & 2.0 \\
S.E & $(4.2)$ & $(83.3)$ & $(11.2)$ & $(0.8)$ \\
M iriápodos & 43.0 & 1.1 & 27.0 & 2.1 \\
S.E & $(2.8)$ & $(72.9)$ & $(104.5)$ & $(1.6)$ \\
Otros & 145.0 & 4.5 & 53.0 & 0.5 \\
S.E & $(7.3)$ & $(626.5)$ & $(49.0)$ & $(0.50)$ \\
\hline TOTAL & 1719.0 & 30.8 & 4099.0 & 24.1 \\
S.E & $(75.8)$ & $(1092.6)$ & $(2152)$ & $(10.4)$ \\
\hline
\end{tabular}

\section{TALA DEL BOSQUE}

Se utilizó un bosque secundario aproximadamente de 20 años con el sistema tradicional de preparación del suelo: rozo, tumba y quema para los cultivos de maíz, arroz y caupí. La quema de la biomasa aérea (hojas y troncos) se hizo fuera de la parcela para evitar quema de la malla plástica. 
Para la instalación de las parcelas se cercaron un área de $60 \mathrm{~cm}$ de diámetro. L as mallas fueron introducidas hasta una profundidad de $45 \mathrm{~cm}$ y con un margen de $10 \mathrm{~cm}$ sobre la superficie para evitar la salida y/o entrada de lombrices.

El diseño experimental incluye tres bloques divididos en 108 unidades (Figura 3) tres tratamientos de manejo de residuos, 2 subtratamientos de lombrices. L as parcelas tenían un área de parcela de $202 \mathrm{~m}^{2}$, con un área neta de $0.28 \mathrm{~m}^{2}$ (60 cm de diámetro) de cada unidad experimental. Se cosecharon 6 ciclos de cultivos anuales.

Los tratamientos de residuos fueron:

$C: C$ ontrol sin material vegetal aportado en la superficie

CR: Cultivo con rastrojo de los cultivos

CRV : Con rastrojo y abono verde de leguminosa marcado con $15 \mathrm{~N}$.

LoS 2 subtratamientos de lombrices fueron (con lombrices: $L$, sin lombrices). Para determinar si existe diferencia significativa entre tratamientos y subtratamientos se hizo la Test de Duncan y LSD al $5 \%$.

\section{ELIMINACION DE LOMBRICES}

Inicialmente se hicieron ensayos preliminares con pesticidas (Benomyl) con una dosis de $6.6 \mathrm{~g} \mathrm{~m}^{-2}$; donde no se obtuvo resultados positivos debido al parecer que su efecto es a largo plazo. Con Furadan 4F (Carbofuradan) se obtuvo resultados positivos a corto plazo ( 24 y 48 horas después de su aplicación) con una mortandad de $100 \%$ con una dosis de $0.9 \mathrm{ml} /$ litro de agua. El carbofuradan tiene un efecto residual aproximado de 50 días, después de su aplicación.

\section{MANEJ O DE CENIZAS}

Después de la tala, todo el material vegetal fue ubicado a un costado de la parcela. U na vez seco este material se procedió a la quema, y luego al recojo de la ceniza incluido el carbón para obtener el peso total. Se aplicó $1.6 \mathrm{~T}$ ha-1 de ceniza ( $45 \mathrm{gr}$. por parcela de $0.28 \mathrm{~m}^{2}$ ) en forma directa y uniforme, luego se regó con 5 litros de agua/parcela en forma lenta, simulando una Iluvia. El resto de ceniza fue aplicado en la parcela no experimental.

\section{SIE MBRA}

La siembra de maíz (Zea rnays), arroz (Oryza sativa), y caupí (Vigna unguiculata) se hizo con la metodología del sistema tradicional (tacarpo), a un distanciamiento de 40 y $25 \mathrm{~cm}$ entre hileras y plantas respectivamente. En cada parcela se sembraron dos plantas ( 2 hoyos). 
12

B. Pashanasi, P. Lavelle y J. Alegre

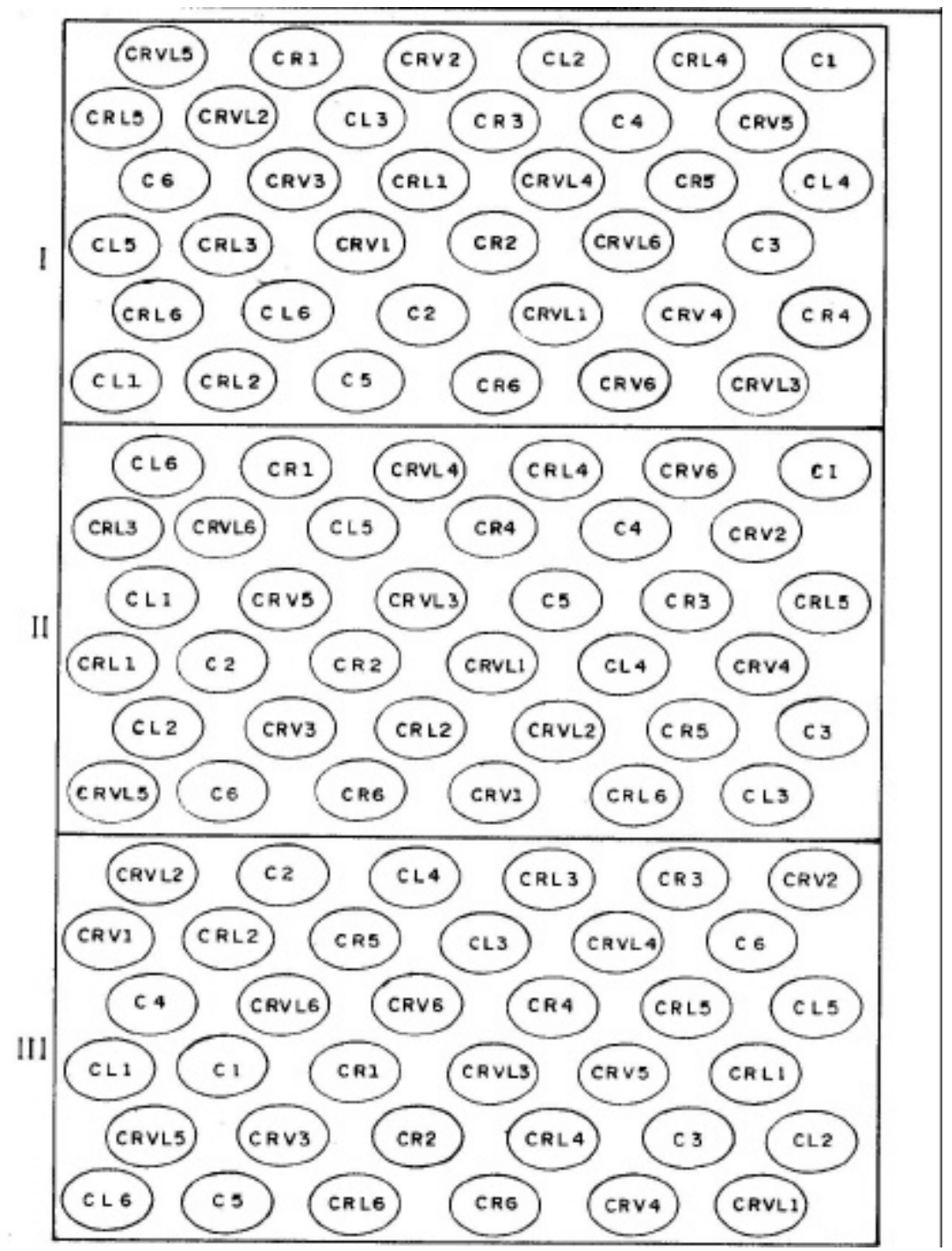

Figura 3. Distribución de los tratamientos en el área experimental $C$ : Control con suelo desnudo; CR: Residuos de cosecha en la superficie del suelo; CRV: Residuos de cosecha + abono verde de leguminosa (centrosema macrocarpum). Todos los tratamientos con L tiene lombrices (pontoscolex corethrurus). LoS números indican el número de cosecha efectuada: 1 (1ra. cosecha), 2 (2da. cosecha), 3 (3ra. cosecha), 4 (4ta. cosecha), 5 (5ta. cosecha) y 6 (6ta. cosecha). 


\section{AMPLIACION DE MATERIAL VEGETAL}

A ntes del inicio de la primera rotación se incorporó rastrojo de arroz (2.5 $\mathrm{T} \mathrm{ha}^{-1}$ peso seco, $70 \mathrm{~g}$ por parcela), con una porción de abono verde de Centrosema macrocarpum marcado con $15 \mathrm{~N}$ a una proporción de $1.8 \mathrm{~T}_{\text {ha }}{ }^{-1}$ peso seco. Los rastrojos fueron depositados en la superficie de la parcela, sin ningún ordenamiento. Al término de cada cosecha, el material restante y residuos de cosecha se dejó para los próximos cultivos. A partir de la segunda rotación se incorporó abono verde de Centrosema macrocarpum no marcado a razón de 2,5 $\mathrm{T}$ ha-1 de peso seco (70 $\mathrm{g}$ por parcela)

\section{INTRODUCCION DE LAS LOMBRICES}

Los cultivos en el laboratorio y el campo de Pontoscolex corethrurus ha demostrado que se adapta bien a las condiciones del suelo, ninguna de las especies nativas tiene capacidad de resistir a la deforestación. En Y urimaguas, corno en la mayor parte de la A mazonía es común encontrar $P$. corethrurus en suelos disturbados de patios, jardines y pasturas. Esta especie es originaria de Guyana, pero tiene una distribución pantropical. La cantidad de Pontoscolex corethrurus inoculada fue de $360 \mathrm{~kg} \mathrm{ha}^{-1}$, peso fresco. La inoculación fue hecha 10 días después de la siembra del primer cultivo. La cantidad utilizada es menor a los ecosistemas de pastizales tradicionales y mejoradas (Lavelle y Pashanasi 1989).

\section{MEDICION DE LOS PRINCIPALES PARAMETROS}

Biomasa de partes aéreas y producción de granos

La producción de granos y rastrojo fue medida en cada cosecha, en todas las unidades experimentales, en forma individual, por planta, por parcela, por unidad de tratamiento y subtratamiento. La biomasa restante fue incorporada a la unidad experimental con excepción de los tratamientos $\mathrm{C}$ y $\mathrm{Cl}$.

Densidad y biomasa de lombrices y raíces

Se usó la metodología TSBF (Tropical Soil and Biology Fertility) (Fig. 4). De cada unidad se sacó 2 cuadrantes para el muestreo de raíces y lombrices por capas de $10 \mathrm{~cm}$ hasta los $20 \mathrm{~cm}$ de profundidad. Luego el suelo fue puesto en bolsas plásticas y llevados al laboratorio para la separación de raíces por elutriación. 


\section{Biomasa microbiana}

En el centro de la unidad se cortó una faja de suelo de $5 \mathrm{~cm}$ de ancho en capas de $10 \mathrm{~cm}$ hasta una profundidad de $20 \mathrm{~cm}$ para los análisis de mineralización y biomasa microbiana. La biomasa microbiana fue determinada por el método de Cahussod, 1990. El N-NH 4 y N-NO $0_{3}$ por el método de Ninhydrin.

\section{Medidas químicas y físicas del suelo}

En cada cosecha se hizo las siguientes evaluaciones: análisis del contenido de nutrientes, densidad aparente, lectura de tensiómetros, infiltración de agua, agregados del suelo (medidos por el método del tamizado en seco de 4 monolitos de $10 \times 10 \times 10 \mathrm{~cm}$ ) (Figura 4). 


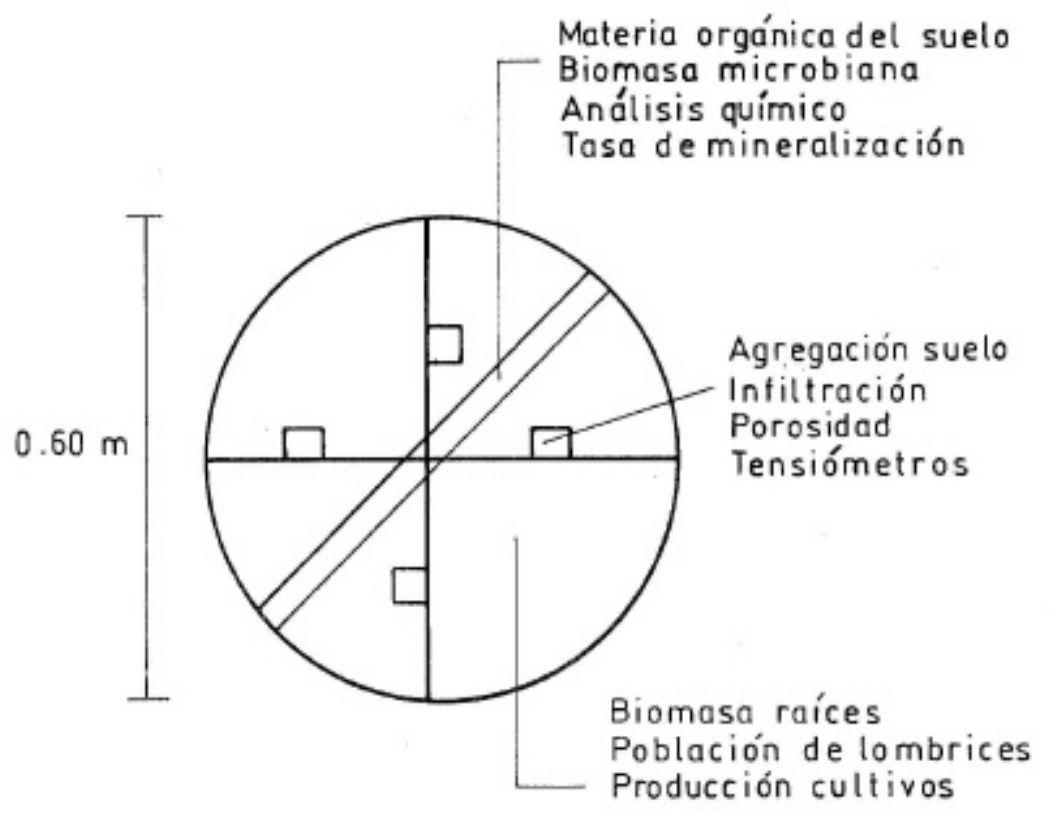

Figura 4. Diseño de muestreo en la unidad experimental en cada cosecha. 


\section{RESULTADOS}

\section{PRODUCCJON DE LAS PLANTAS}

La producción de las plantas se vio afectada por la introducción de lombrices y por la cantidad y calidad de los insumos orgánicos. Los incrementos en la producción de granos debido a la actividad de las lombrices y aplicación de residuos orgánicos fue generalmente mayor que los incrementos en la producción de rastrojo y raíces (Figura 5)

\section{PRODUCCJON DE GRANOS}

La producción se mantuvo en niveles relativamente altos en comparación a los rendimientos locales. En los tratamientos con aplicación de abono verde de leguminosa e inoculación de lombrices, la producción promedio fue $1.62 \mathrm{~T}^{\text {ha-1 }}{ }^{-1}$ para 6 cultivos sucesivos o sea $112 \%$ más que en el control sin lombrices 0 aplicación de residuos orgánicos $\left(0.77 \mathrm{~T} \mathrm{ha}{ }^{-1}\right)$. 

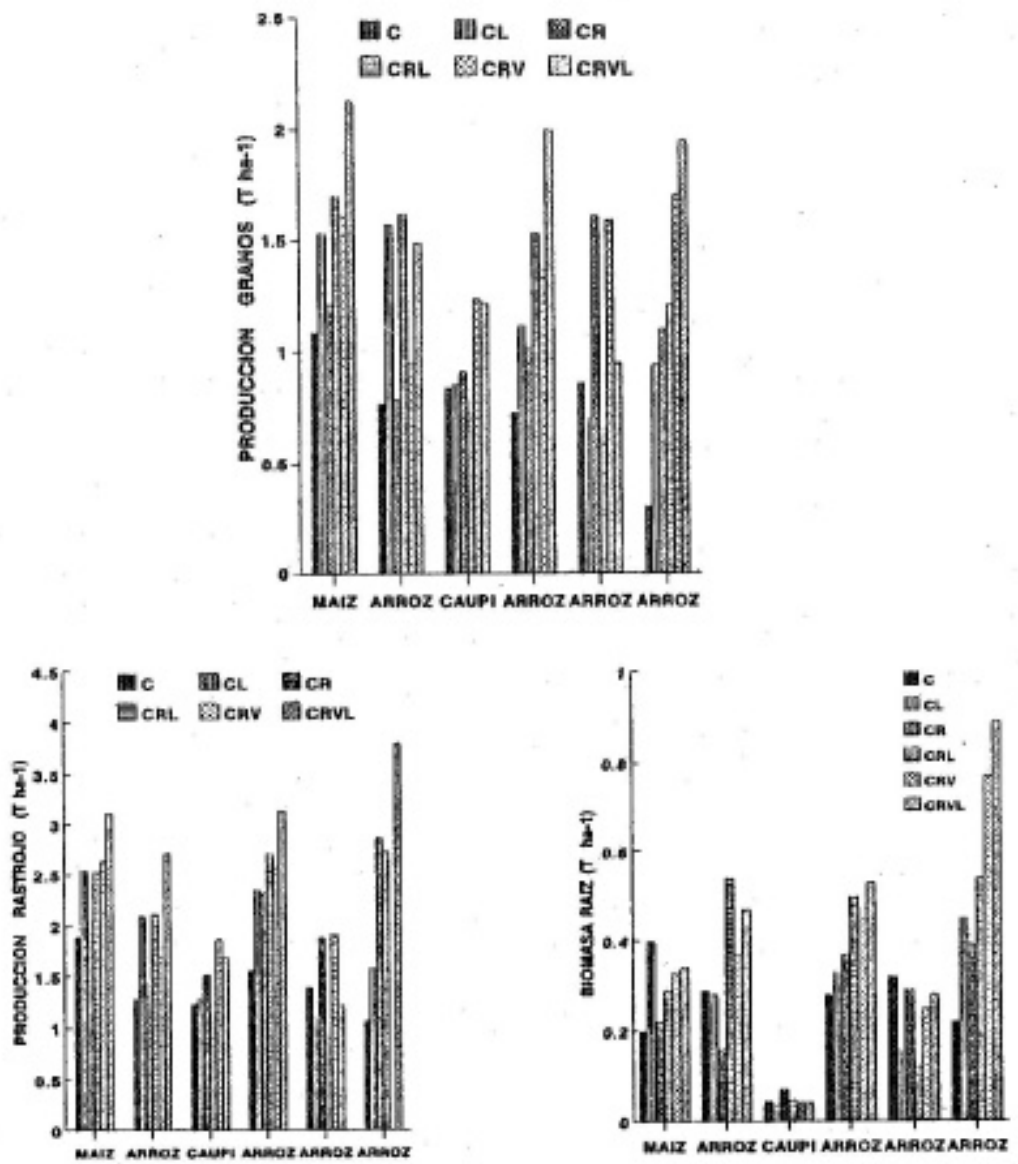

Figura 5. Producción de granos, rastrojos y raíces durante 6 periodos consecutivos de cosecha. 
El crecimiento también fue rápido con la presencia de lombrices y la maduración completa fue obtenida 2 semanas antes en los tratamientos con lombrices.

En el primer cultivo, la producción de maíz varió de 1.1 a 2.11 ha-1. No hubo efecto significativo entre tratamientos, incrementándose la producción con la cantidad y calidad de mulch incorporado en el suelo. En la comparación entre subtratamientos se encontró efecto significativo de la inoculación de lombrices. Los rendimientos se incrementaron en $31.5 \%, 39.3 \%$ y $40.4 \%$ respectivamente en los tratamientos CRV, CR y C. El incremento de la producción fue mucho mayor cuando los tratamientos recibieron más insumos orgánicos. Similar tendencia fue observada en la producción de rastrojo y raíces (Cuadro 2).

\section{Cuadro 2}

Producción de granos, rastrojo y raíces $\left(\mathrm{T} \mathrm{ha}^{-1}\right)$ en seis rotaciones de cultivo con y sin lombrices.
$\mathrm{C}$
CL
$\mathrm{CR}$
CRL
CRV CRVL

1er cultivo

MAIZ

\begin{tabular}{lllllll} 
Granos & 1.09 & 1.53 & 1.22 & 1.70 & 1.62 & 2.13 \\
Rastrojo & 1.89 & 2.55 & 2.05 & 2.52 & 2.64 & \\
Raíces & 0.20 & 0.40 & 0.22 & 0.44 & 0.33 & 0.34 \\
\hline
\end{tabular}

2do cultivo

ARROZ

\begin{tabular}{lllllll} 
Granos & 0.77 & 1.57 & 0.78 & 1.62 & 0.95 & 1.49 \\
Rastrojo & 1.28 & 2.09 & 1.31 & 2.13 & 1.80 & 2.71 \\
Raíces & 0.29 & 0.29 & 0.16 & 0.54 & 0.37 & 0.47 \\
\hline
\end{tabular}

3er cultivo

\section{CAUPí}

Granos

Rastrojo

0.84

0.85

0.91

0.78

1.24

1.22

Raíces

1.56

2.35

2.32

2.71

2.60

3.14

0.05

0.04

0.07

0.05

0.04

0.04 
Continúa

Cuadro 2

Producción de granos rastrojo y raíces ( 1 ha-1) en seis rotaciones de cultivo con y sin lombrices.

\begin{tabular}{lllllll}
\hline $\mathrm{C}$ & $\mathrm{CL}$ & $\mathrm{CR}$ & $\mathrm{CRL}$ & $\mathrm{CRV}$ & $\mathrm{CRVL}$ \\
\hline
\end{tabular}

4to. cultivo

\section{ARROZ}

\begin{tabular}{lllllll} 
Granos & 0.73 & 1.12 & 1.02 & 1.53 & 1.39 & 2.00 \\
Rastrojo & 1.56 & 2.35 & 2.32 & 2.71 & 2.60 & 3.14 \\
Raíces & 0.28 & 0.33 & 0.37 & 0.50 & 0.47 & 0.53 \\
\hline
\end{tabular}

5to. cultivo

\section{ARROZ}

$\begin{array}{lllllll}\text { Granos } & 0.86 & 0.71 & 1.61 & 0.66 & 1.59 & 0.95 \\ \text { Rastrojo } & 1.39 & 1.09 & 1.88 & 0.98 & 1.92 & 1.23 \\ \text { Raíces } & 0.32 & 0.16 & 0.29 & 0.15 & 0.25 & 0.28\end{array}$

6to. cultivo

$\begin{array}{lllllll}\text { ARR OZ } & & & & & & \\ \text { Granos } & 0.30 & 0.94 & 1.10 & 1.21 & 1.70 & 1.95 \\ \text { Rastrojo } & 0.98 & 1.82 & 2.64 & 2.82 & 3.22 & 4.08 \\ \text { Raíces } & 0.22 & 0.45 & 0.39 & 0.54 & 0.77 & 0.89\end{array}$

En la segunda cosecha, la producción de arroz estuvo en un rango de 0.77 a $1.491 \mathrm{ha}^{-1}$. El efecto de los residuos orgánicos no fue significativo, contrario a la inoculación de lombrices con incrementos de 103.9, 107.7 y $56.8 \%$ para los tratamientos C, CR y CRV respectivamente. Otra vez el efecto de las lombrices fue menos marcado en los tratamientos con menos insumo orgánico. La producción obtenida en CRV con presencia de lombrices fue alto en comparación a los rendimientos locales y cerca a la producción obtenida en suelos aluviales ricos en nutrientes y con tecnología tradicional.

Contrario a las dos primeras cosechas, el caupí como tercera rotación de cultivo tuvo rendimientos entre 0.84 a 1.221 ha $^{-1}$ donde no hubo efecto significativo de la presencia de lombrices sobre la producción. Sólo hubo un incremento de $1.2 \%$, un decrecimiento de -14.1 , y un incremento de $1.4 \%$ para los tratamientos CRV, CR y $C$ respectivamente. Sin embargo, fue observada un efecto altamente significativo sobre la producción de granos y rastrojo en los tratamientos con CRV que en los tratamientos con CR. La producción se 
mantuvo en niveles relativamente en comparación a los rendimientos locales (Cuadro 2).

En la cuarta cosecha con arroz se obtuvo rendimientos que oscilaron entre 0.73 a $2.00 \mathrm{~T} \mathrm{ha}^{-1}$. Se encontró diferencia significativa entre tratamientos en la producción de granos. Los mayores rendimientos se obtuvieron en los tratamientos que recibieron insumos orgánicos en comparación a suelo desnudo. Hubo efecto significativo de la presencia de lombrices en la producción de granos con incrementos de $43.9 \% ; 50.0 \%$; y $53.4 \%$ respectivamente para los tratamientos CRV; CR y C (Figura 5). Estos rendimientos son relativamente al tos con relación a los rendimientos locales de la variedad tradicional "Carolino'. Similar tendencia fue observado en rastrojo y raíces.

En la quinta rotación con arroz, se obtuvo rendimientos de granos en un rango de 0.86 a $1.61 \mathrm{~T}^{\mathrm{h}}{ }^{-1}$. Hay diferencia altamente significativa entre tratamientos. Siendo la mayor producción en los tratamientos con rastrojo + abono verde y la más baja en el tratamiento de suelo desnudo. No hubo efecto de la presencia de lombrices, al contrario hubo un decrecimiento en la producción de granos de -17.4,

-59.0y $40.3 \%$ para los tratamientos C, CR, y CRV respectivamente. Este decrecimiento quizás se deba a la fuerte competencia entre lombrices y planta por agua en el período vegetativo del arroz. Similar tendencia fue observada en rastrojo y raíces (Figura 5).

En la sexta cosecha de arroz, con buena precipitación en las ¿pocas críticas de requerimiento de agua, se obtuvo rendimientos que oscilan entre 0.30 a $1.95 \mathrm{~T}$ ha-2. Hubo una diferencia al tamente significativa entre tratamientos, obteniéndose el mayor rendimiento en el tratamiento CRV. A sí mismo tuvo efectos positivos la presencia de lombrices sobre la producción en los tratamientos C, CR y CRV con incrementos de $213.3,10.0$ y 1.95 respectivamente (Cuadro 2 ).

$L$ a inoculación de lombrices tuvo efectos significativamente positivos sobre la producción de granos en el primer cultivo (maíz $+37 \%$ en promedio), 2 do (arroz, $+88 \%$ en promedio), 4 to y 6 to ( 50 y $52 \%$ en promedio); no hubo efecto en la tercera cosecha (caupí, $-5 \%$ no significativo) y un efecto negativo en la quinta cosecha (arroz, - $43 \%$ ) (Figura 6). 


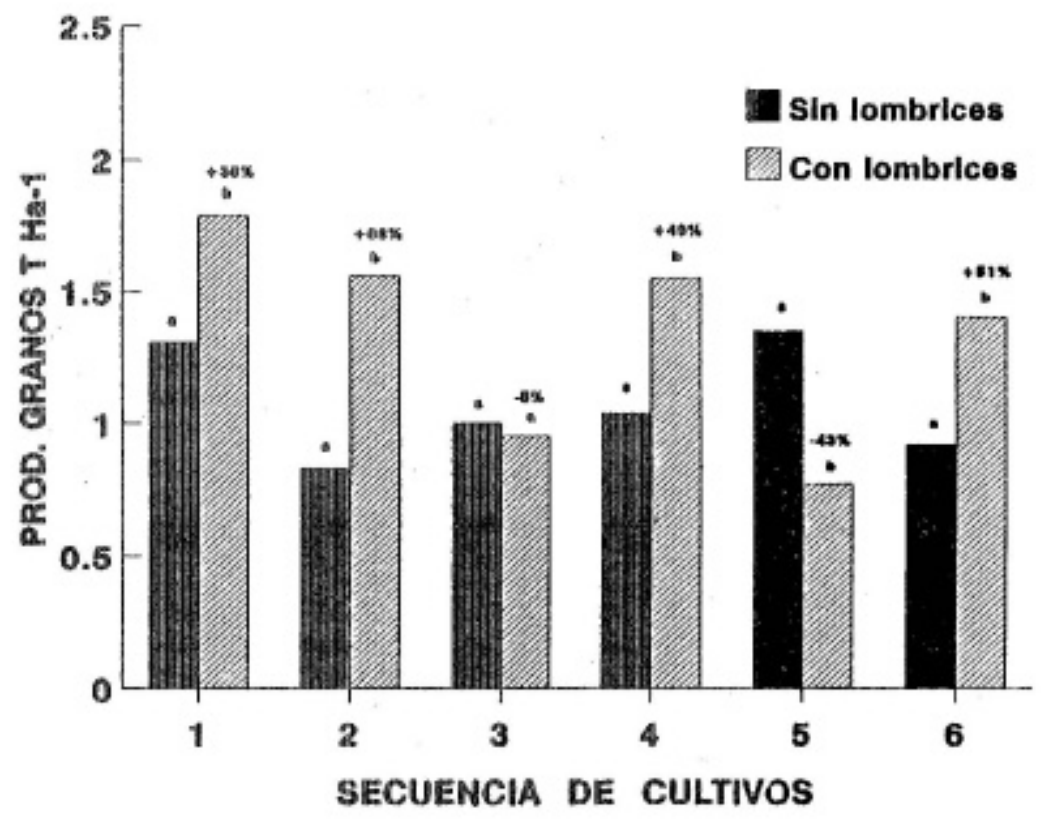

Figura 6. Efecto de la actividad de las lombrices sobre la producción de granos sin considerar los tratamientos orgánicos en 6 cosechas consecutivas.

El efecto de las lombrices fue significativamente diferente dependiendo del tratamiento orgánico: el incremento promedio de producción de granos fue $46 \%$ en el control y en el tratamiento CRV (rastrojo + abono verde) 15\% y sólo $13 \%$ en el tratamiento CR (rastrojo) (Figura 7).

Estos resultados muestran que la interacción entre la calidad de los residuos orgánicos, la materia orgánica aprovechable y la actividad de las lombrices pueden eventualmente resultar en efectos diferentes sobre el crecimiento de la planta. 


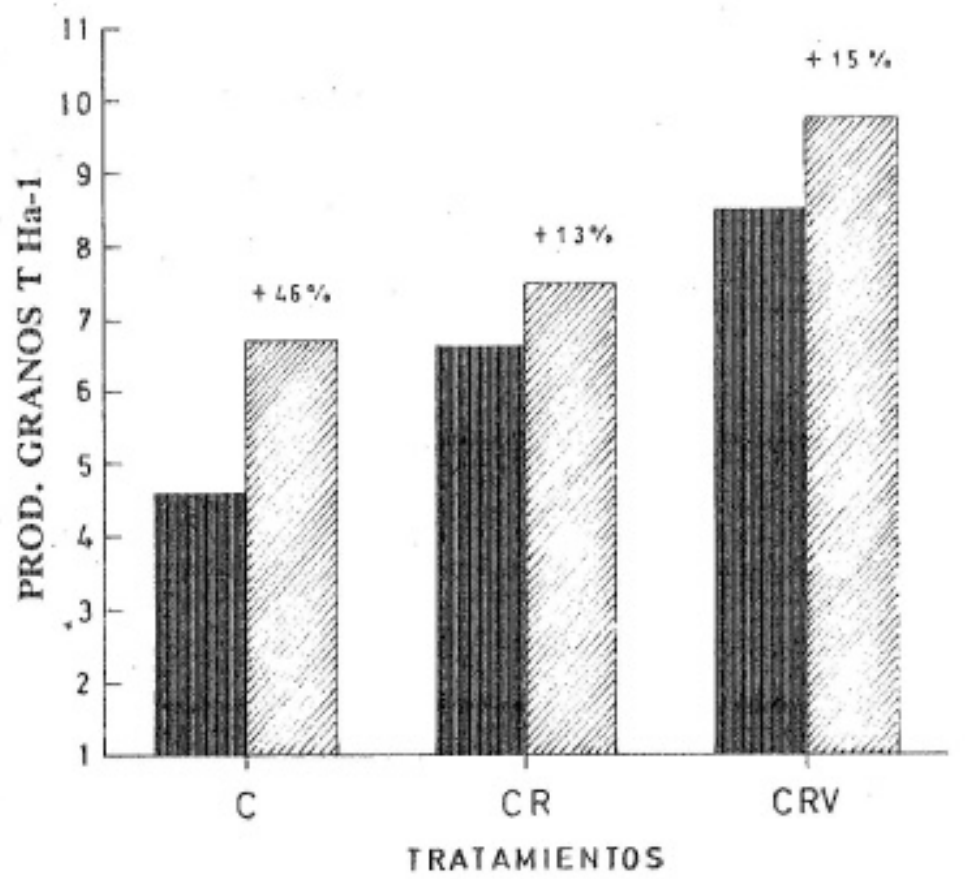

Figura 7. E fecto de la actividad de las lombrices en los diferentes tratamientos con 6 cosechas consecutivas.

Como se esperaba, los tratamientos orgánicos también tienen efectos sobre el crecimiento de las plantas con incrementos de 26 y $62 \%$ de producción de granos en los tratamientos que recibieron rastrojo o rastrojo + abono verde de leguminosa comparado al tratamiento control (Figura 8). 


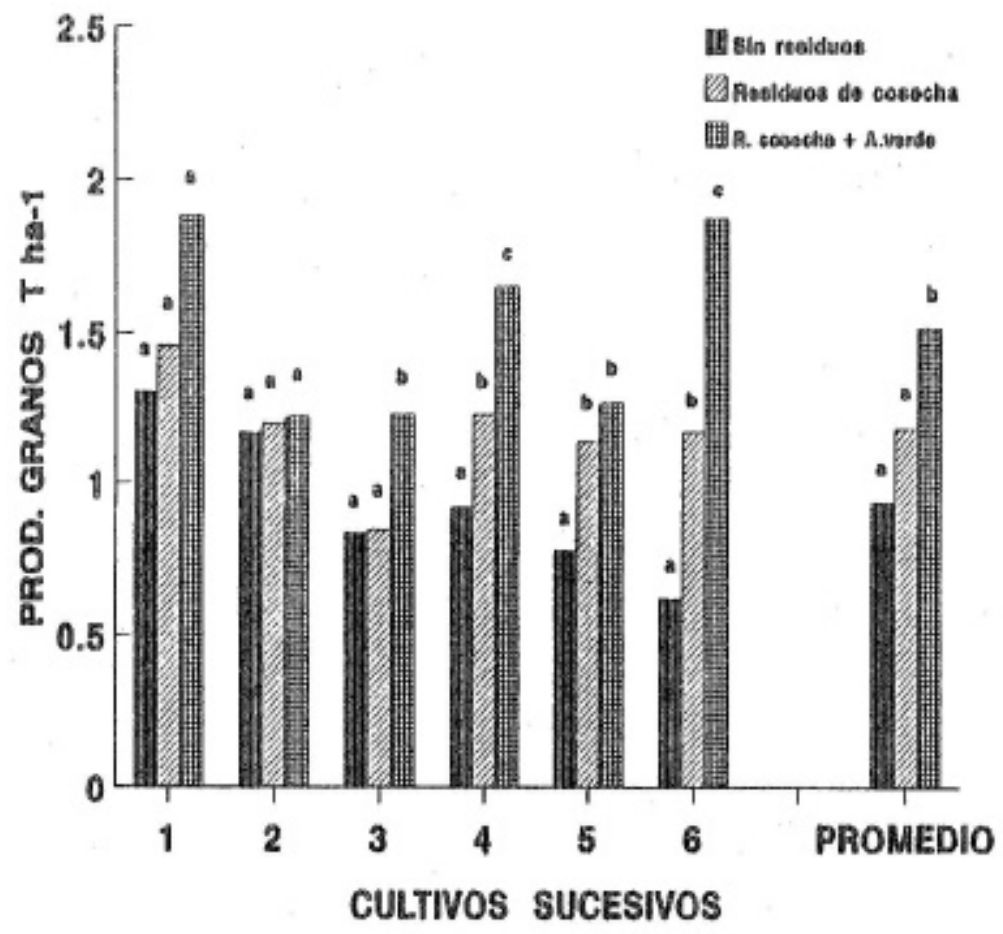

Figura 8. Efecto de los insumos orgánicos en la producción de granos en 6 cosechas sucesivas, sin considerar la actividad de las lombrices.

\section{DINAMICA DE LA POBLACION DE LOMBRICES}

La población de lombrices inoculadas se establecieron satisfactoriamente. L a biomasa tuvo un decrecimiento ligero en la primera cosecha. Sin embargo, la población fue compuesta principalmente de individuos jóvenes y con presencia de gran número de capullos. El mínimo valor fue observado en el tratamiento de suelo desnudo, pero la diferencia no fue significativa (Figura 9). 


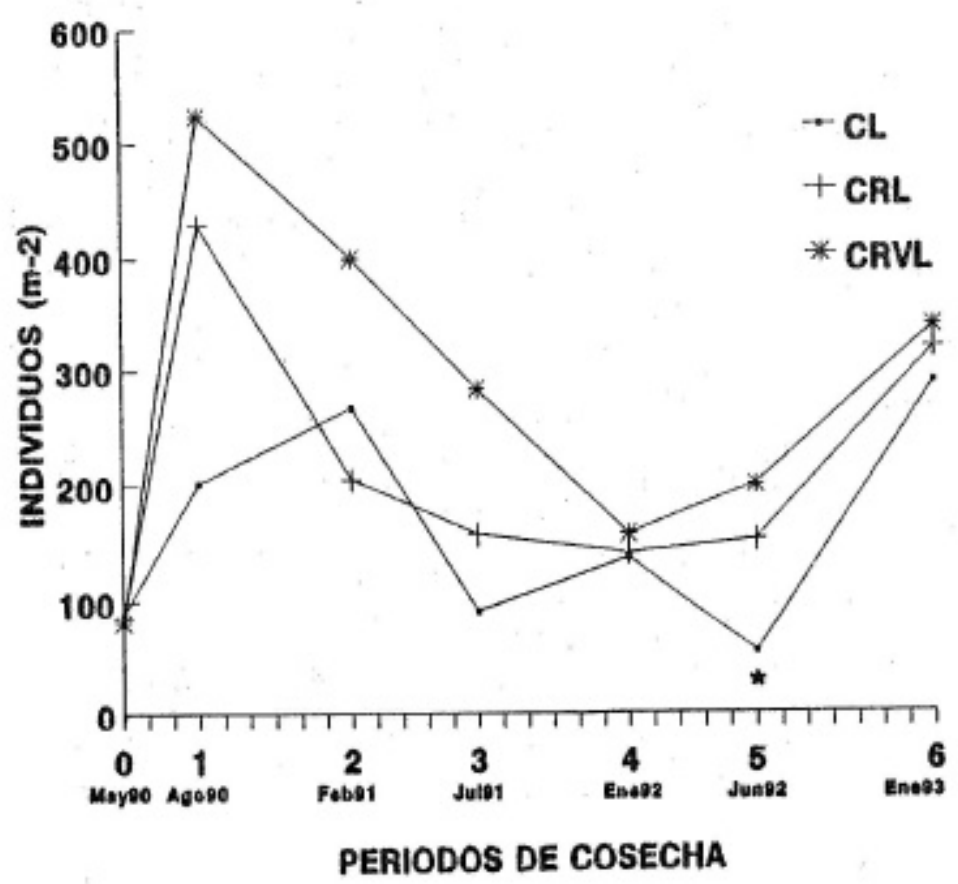

Figura 9. Cambios en la densidad de lombrices durante 6 ciclos sucesivos de cosecha. Datos con * indica diferencia significativa con el tiempo.

En la segunda cosecha la biomasa tuvo un incremento en todos los tratamientos, la diferencia sólo fue significativa en los tratamientos que recibieron residuos de cosecha y abono verde de leguminosa. La biomasa alcanzó $81.4 \mathrm{~g}$ de peso fresco, es decir 2.3 veces más que la biomasa original introducida (Figura 10)

No hubo diferencia significativa entre tratamientos en biomasa y densidad de lombrices en la tercera cosecha. Hubo una disminución en la población de lombrices debido a la escasez de agua en el suelo durante ese período de cultivo (junio-julio), manteniéndose la biomasa muy similar a la segunda cosecha. En la cuarta cosecha hubo una disminución en la población y biomasa de lombrices con 
relación a las cosechas anteriores, esto debido a la prolongada sequía en la zona que afectó la reproducción y crecimiento de las lombrices.

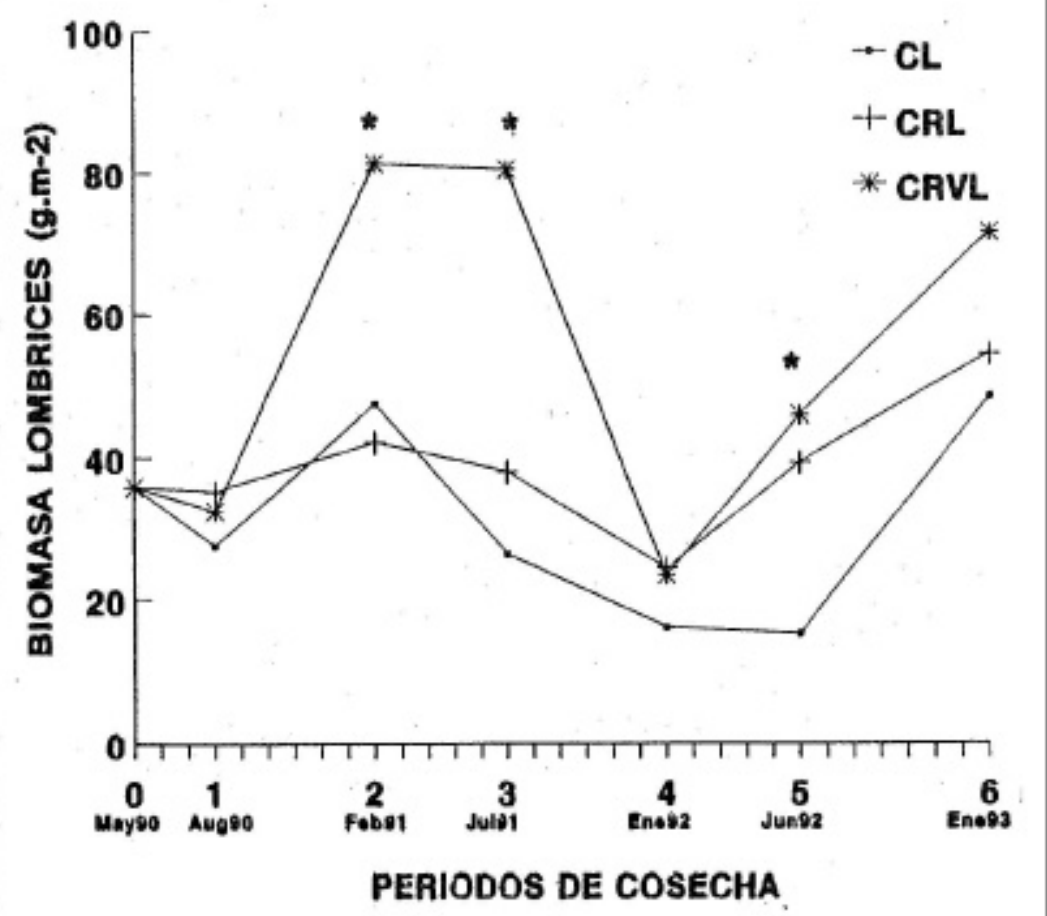

Figura 10. Cambios en la biomasa de lombrices durante 6 ciclos sucesivos de cosecha. Datos * indican diferencia significativa en el tiempo indicado.

En la quinta cosecha hubo diferencia significativa entre tratamientos en densidad y biomasa; siendo la población y biomasa de 2 a 3 veces más en los tratamientos con insumos orgánicos en comparación al suelo desnudo.

En la sexta cosecha no hubo diferencia significativa entre tratamientos en población y biomasa de lombrices. El incremento de densidad y biomasa se debe 
a la mayor precipitación caída en ese período de cultivo. Después de 6 cosechas consecutivas en un lapso de 33 meses el incremento de densidad fue de 224, 271 y

$313 \%$ para los tratamientos C, CR y CRV . La biomasa se incrementó en un $98 \%$ al final de nuestro experimento en el tratamiento CRV (Cuadro 3).

\section{Cuadro 3}

Densidad y biomasa (g peso fresco $\mathrm{m}^{-2}$ ) de Pontoscolex corethrurus en los tratamientos C (sin residuo orgánico en la superficie del suelo), CR (rastrojo) y CRV (rastrojo + abono verde de leguminosa)

\begin{tabular}{|c|c|c|c|c|c|c|c|}
\hline & Inic. & M aíz & Arroz & Caupí & Arroz & Arroz & Arroz \\
\hline \multicolumn{8}{|c|}{ Biomasa } \\
\hline \multicolumn{8}{|c|}{$\mathrm{g} \mathrm{m}^{-2}$} \\
\hline$C L$ & 36.0 & $27.5 \mathrm{a}$ & $47.4 \mathrm{a}$ & $26.0 \mathrm{a}$ & $16.2 \mathrm{a}$ & $15.3 \mathrm{a}$ & $48.3 a$ \\
\hline CRL & 36.0 & $35.3 \mathrm{a}$ & $42.1 \mathrm{a}$ & $38.1 \mathrm{a}$ & $24.3 \mathrm{a}$ & $39.3 \mathrm{ab}$ & $54.3 a$ \\
\hline CRVL & 36.0 & $32.5 \mathrm{a}$ & $81.4 \mathrm{~b}$ & $80.5 \mathrm{a}$ & $23.4 \mathrm{a}$ & $45.8 \mathrm{~b}$ & $71.4 a$ \\
\hline \multicolumn{8}{|c|}{$\begin{array}{l}\text { Densidad } \\
\mathrm{m}^{-2}\end{array}$} \\
\hline$\overline{C R}$ & 89.0 & $202.3 a$ & $266.7 \mathrm{a}$ & $90.7 a$ & $137.3 a$ & $55.0 \mathrm{a}$ & $288.7 a$ \\
\hline$C R L$ & 86.0 & $428.7 a$ & $205.0 \mathrm{a}$ & 157.3a & $140.6 a$ & $152.3 \mathrm{~b}$ & $319.3 \mathrm{a}$ \\
\hline CRVL & 82.0 & $523.7 a$ & $400.0 \mathrm{a}$ & 283.3a & 157.3a & $200.0 \mathrm{~b}$ & $338.3 \mathrm{a}$ \\
\hline
\end{tabular}

$\mathrm{L}=\mathrm{L}$ ombrices

\section{CAMBIOS EN LA ESTRUCTURA FISICA DEL SUELO}

La estructura física del suelo se Vio afectada por la actividad de las lombrices. Pontoscolex corethrurus puede ingerir diariamente 6 veces más que su peso dependiendo de su tamaño y condición del suelo (Lavelle, et al 1987). A sumiendo una tasa promedio al consumo de $3 \mathrm{gr}$ de suelo seco por $\mathrm{g}^{-1}$ de biomasa fresco, y una conversión estimada de 300 días por año de actividad, uno puede estimar la cantidad de suelo ingerido por las lombrices durante nuestro experimento. Los valores estimados son desde casi $90 \mathrm{~T}^{-1}$ en $C L$ a $150 \mathrm{~T}^{-1}$ en CRL y $220 \mathrm{~T}^{\mathrm{h}} \mathrm{h}^{-1}$ en CRVL; ellos incrementaron significativamente la cantidad de insumos orgánicos. 
Macroagregación del Suelo

El suelo ingerido es expulsado como turriculos después de la ingestión. LoS turrículos están depositados en la mayor parte dentro del suelo, en la parte superior de los $10 \mathrm{~cm}$. del suelo. Las lombrices generalmente ingieren agregados mas pequeños que el diámetro de su boca (Blanchart, 1990). En contraste, los turrículos son agregados grandes de diversos $\mathrm{mm}$ de diámetro. Como consecuencia de la actividad de alimentación de las lombrices, los pequeños agregados son transformados en más grandes. Después de algún tiempo, este proceso induce cambios significativos en la agregación del suelo que abarca el perfil (Figura 11).

La agregación de suelo ha cambiado significativamente con el tiempo. En los tratamientos sin lombrices, la proporción de pequeños agregados $(<0.5 \mathrm{~mm}$ ) se ha incrementado con el tiempo (desde 32.5 a $35.4 \%$ ) a expensas de agregados del tamaño intermedio (2-10 mm) cuya relativa abundancia decrece de 40.8 a $39.2 \%$. Con la presencia de lombrices, la muestra fue significativamente diferente: la proporción de pequeños agregados decreció desde 30.7 a 27.4. Considerando la proporción de grandes agregados $(>1 \mathrm{~cm}$ ) ha incrementado de 25.5 a $31.3 \%$. 

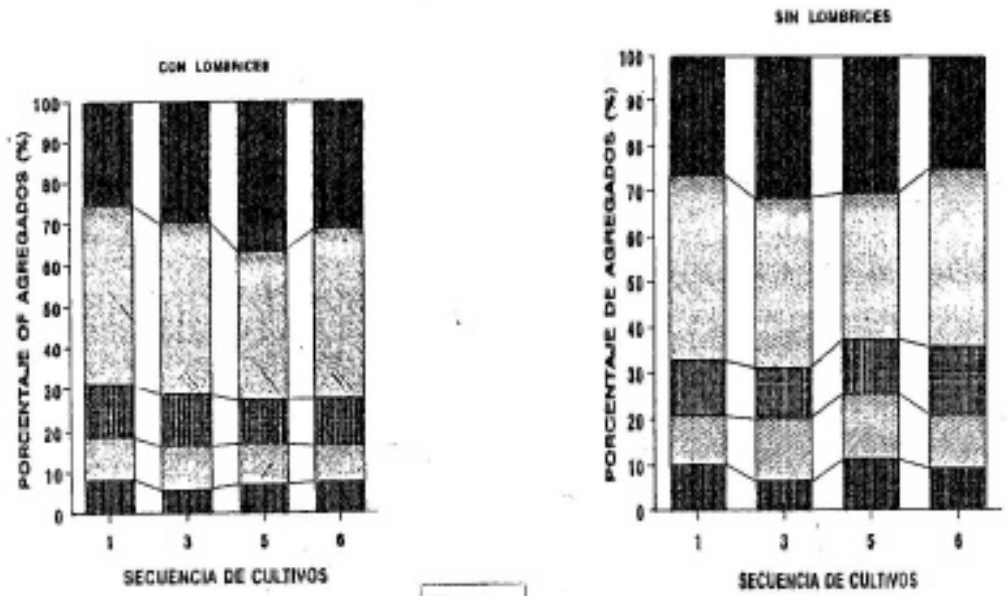

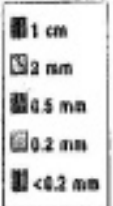

Figura 11. Cambios de agregación del suelo en el experimento.

\section{Densidad aparente}

Algunos cambios en la agregación ha resultado en modificaciones significativas de todos los otros parámetros de la estructura física del suelo. La densidad aparente en la capa de $10 \mathrm{~cm}$ de suelo fue significativamente más al to en los tratamientos con lombrices que los tratamientos sin lombrices (Cuadro 5).

Después de un constante incremento durante los tres primeros cultivos, la densidad aparente fue estabilizada en 1.22 en los tratamientos con lombrices luego de 6 cosechas. En los tratamientos sin lombriz, la densidad aparente ha fluctuado desde 1.05 a 1.16 y no fue significativamente diferente al bosque original (Figura 12). 


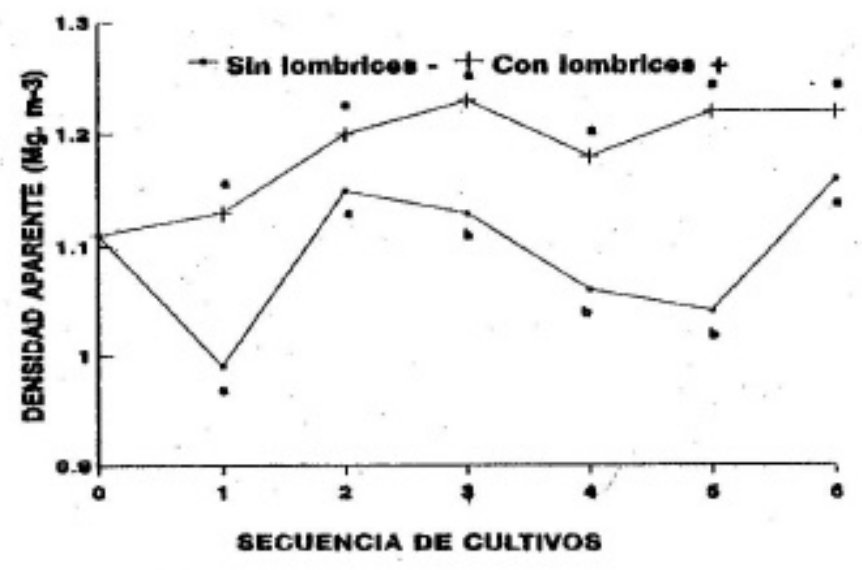

Figura 12. Cambios de densidad aparente con el tiempo. Datos con diferentes letras indican diferencia significativa. 


\section{Cuadro 4}

Densidad aparente (DA) y humedad del suelo $(H)$ de $0-5 \mathrm{~cm}$. de profundidad después de 6 cosechas consecutivas (maízarroz-caupí-arroz-arroz-arroz)

\begin{tabular}{|c|c|c|c|c|c|c|c|c|c|c|c|c|c|c|c|}
\hline \multirow[t]{2}{*}{ Trat. } & \multicolumn{2}{|c|}{ Inicial } & \multicolumn{2}{|c|}{ M aíz } & \multicolumn{2}{|c|}{ A rroz } & \multicolumn{2}{|c|}{ Caupí } & \multicolumn{2}{|c|}{ Arroz } & \multicolumn{2}{|c|}{ Arroz } & \multicolumn{2}{|c|}{ Arroz } & \multirow{2}{*}{$\begin{array}{l}\text { X DA } \\
\text { (6cultivos) }\end{array}$} \\
\hline & DA & $\mathrm{H}$ & DA & $\mathrm{H}$ & DA & $\mathrm{H}$ & DA & $\mathrm{H}$ & DA & $\mathrm{H}$ & DA & $\mathrm{H}$ & DA & $\mathrm{H}$ & \\
\hline & $\mathrm{g} \mathrm{cm}^{-3}$ & $\%$ & $\mathrm{~g} \mathrm{~cm}^{-3}$ & $\%$ & $\mathrm{~g} \mathrm{~cm}^{-3}$ & $\%$ & $\mathrm{~g} \mathrm{~cm}^{-3}$ & $\%$ & $\mathrm{~g} \mathrm{~cm}^{-3}$ & $\%$ & $\mathrm{~g} \mathrm{~cm}^{-3}$ & $\%$ & $\mathrm{~g} \mathrm{~cm}^{-3}$ & $\%$ & $\mathrm{~g} \mathrm{~cm}^{-3}$ \\
\hline C & 1.10. & 26.0 & 0.96 & 31.4 & 1.12 & 24.9 & 1.17 & 24.2 & 1.12 & 18.2 & 1.06 & 19.9 & 1.16 & 21.3 & 1.10 \\
\hline$C L$ & 1.10 & 26.0 & 1.09 & 22.5 & 1.26 & 29.3 & 1.31 & 24.0 & 1.24 & 20.7 & 1.22 & 22.1 & 1.26 & 15.8 & 1.21 \\
\hline$C R$ & 1.10 & 26.0 & 0.99 & 29.5 & 1.13 & 27.3 & 1.13 & 20.6 & 1.04 & 22.4 & 0.98 & 21.7 & 1.09 & 18.0 & 1.07 \\
\hline$C R L$ & 1.10 & 26.0 & 1.09 & 23.3 & 1.15 & 26.7 & 1.17 & 22.2 & 1.13 & 20.2 & 1.30 & 22.3 & 1.18 & 22.6 & 1.16 \\
\hline CRV & 1.10 & 26.0 & 1.04 & 28.8 & 1.19 & 25.7 & 1.09 & 22.5 & 1.02 & 23.6 & 1.06 & 17.6 & 1.24 & 19.5 & 1.10 \\
\hline CRVL & 1.10 & 26.0 & 1.21 & 24.5 & 1.20 & 26.1 & 1.20 & 19.1 & 1.18 & 17.2 & 1.15 & 19.9 & 1.24 & 19.9 & 1.18 \\
\hline$x$ & 1.10 & 26.0 & 1.06 & 26.6 & 1.18 & 26.7 & 1.18 & 22.1 & 1.12 & 20.4 & 1.13 & 20.6 & 1.20 & 19.5 & \\
\hline
\end{tabular}




\section{Infiltración de agua}

La tasa de infiltración ha decrecido significativamente con el tiempo en todos los tratamientos (Figura 13). Esta reducción fue más pronunciada para los tratamientos con lombrices inicialmente.

En la quinta cosecha, la tasa de infiltración se ha incrementado en los tratamientos con lombriz, llegando a valores similares a los tratamientos sin lombrices. Pero en la sexta cosecha se conserva la tendencia de las cosechas anteriores. Los tratamientos sin residuos orgánicos tienen menor tasa de infiltración en todos los tiempos.

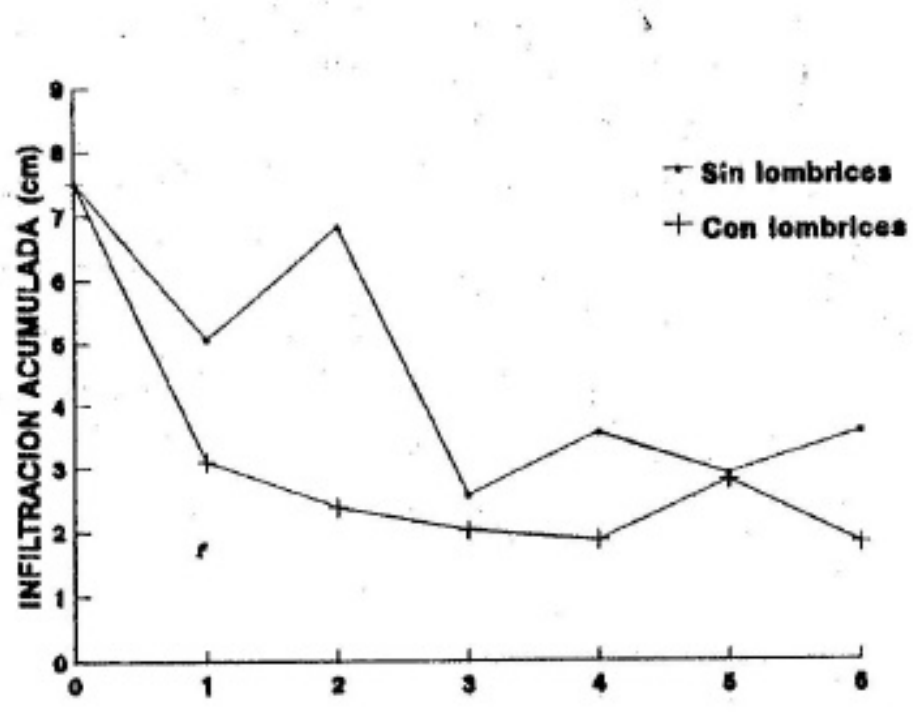

Figura 13. Cambios de infiltración con el tiempo. Datos con diferentes letras indica diferencia significativa 
Regímenes de agua

El régimen de agua de los suelos fue significativamente afectado por la actividad de las lombrices, pero no por los tratamientos orgánicos (Figura 14). En los períodos con menor precipitación la humedad del suelo fue menor en los tratamientos con lombrices. Esto afectó la floración y llenado de granos de los cultivos, y durante estos ciclos la demanda de agua por las plantas es mayor. Durante los períodos de cosecha del segundo y tercer cultivo (Figura 14 a, b), la tensión del suelo fue menor que la capacidad de campo durante todo el ciclo vegetativo del cultivo. Durante las últimas semanas antes de la cosecha del quinto cultivo las tensiones del suelo fueron mayores que la capacidad de campo para el tratamiento con lombrices (Figura $14 \mathrm{c}$ ). Durante los períodos lluviosos la humedad fue muchas veces mayor en los tratamientos con lombrices que en los tratamientos sin lombrices. Esta mayor humedad prolongó un decrecimiento de la infiltración (Cuadro 5). 

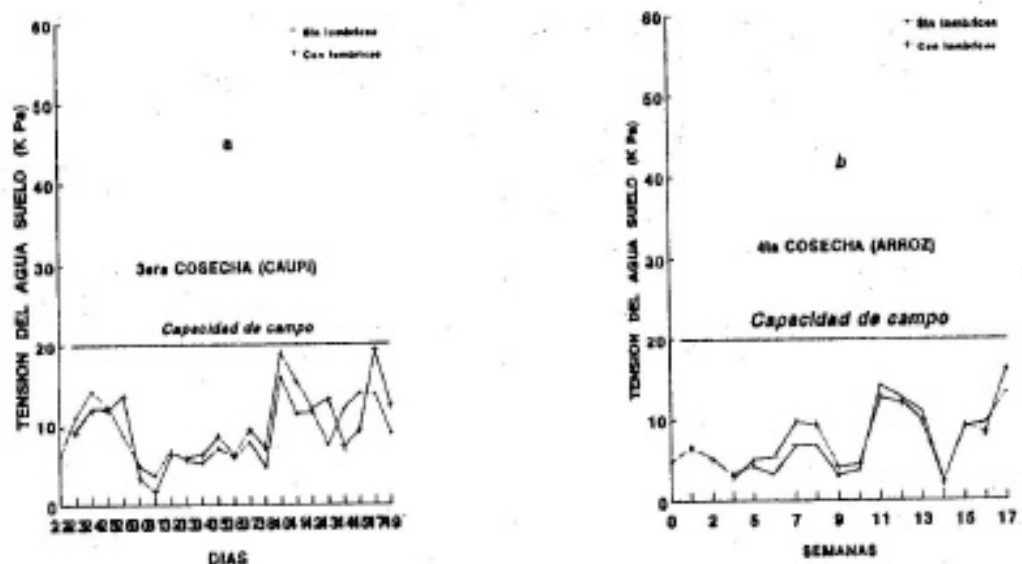

Dus

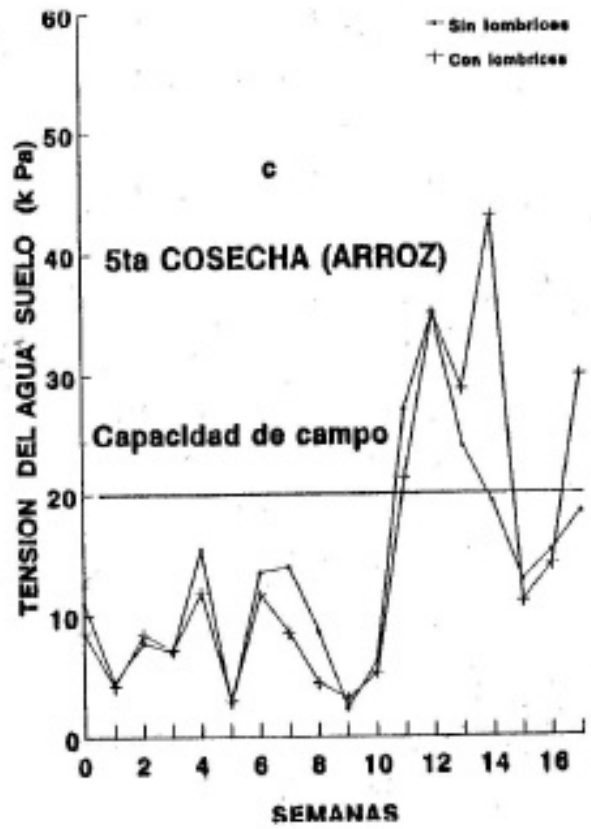

Figura 14. Cambios de tensión del agua en el suelo a la profundidad de 0-10 $\mathrm{cm}$ durante la tercera, cuarta y quinta cosecha. 


\section{Cuadro 5}

Densidad aparente (DA) y humedad del suelo $(H)$ de $0-5 \mathrm{~cm}$. de profundidad después de 6 cosechas consecutivas (maíz-arroz-caupí-arroz-arroz-arroz)

\begin{tabular}{|c|c|c|c|c|c|c|c|c|c|c|c|c|c|c|c|c|}
\hline \multirow[t]{2}{*}{ Trat. } & \multicolumn{2}{|c|}{ Inicial } & \multicolumn{2}{|c|}{ M aíz } & \multicolumn{2}{|c|}{ Arroz } & \multicolumn{2}{|l|}{ Caupí } & \multicolumn{2}{|c|}{ Arroz } & \multicolumn{2}{|c|}{ Arroz } & \multicolumn{2}{|c|}{ Arroz } & \multicolumn{2}{|c|}{$X$ cultivo } \\
\hline & I & S & I & $\mathrm{S}$ & I & $\mathrm{S}$ & I & $\mathrm{S}$ & I & $\mathrm{S}$ & I & $\mathrm{S}$ & 1 & $\mathrm{~S}$ & I & $\mathrm{S}$ \\
\hline $\mathrm{cm} \mathrm{t}^{-1 /}$ & \multicolumn{2}{|c|}{$\mathrm{cm} \mathrm{cm} \mathrm{t} \mathrm{t}^{-1 / 2}$} & \multicolumn{3}{|c|}{$\mathrm{cm} \mathrm{cm} \mathrm{t}^{-1 / 2}$} & \multicolumn{2}{|c|}{$\mathrm{cm} \mathrm{cm} \mathrm{t}-1 / 2$} & \multicolumn{2}{|c|}{$\mathrm{cm} \mathrm{cm} \mathrm{t} \mathrm{t}^{-1 / 2}$} & \multicolumn{2}{|c|}{$\mathrm{cm} \mathrm{cm} \mathrm{t}^{-1 / 2}$} & \multicolumn{2}{|c|}{$\mathrm{cm} \mathrm{cm} \mathrm{t}^{-1 / 2}$} & \multicolumn{2}{|c|}{$\mathrm{cm} \mathrm{cm} \mathrm{t} \mathrm{cm}^{-1 / 2}$} & $\mathrm{~cm}$ \\
\hline C & 7.1 & 0.69 & 3.0 & 0.27 & 5.5 & 0.53 & 1.9 & 0.20 & 3.0 & 0.26 & 2.5 & 0.26 & 2.8 & 0.28 & 3.7 & 0.36 \\
\hline$C L$ & 1.1 & 0.69 & 5.2 & 0.52 & 1.5 & 0.13 & 1.1 & 0.10 & 1.5 & 0.23 & 2.5 & 0.23 & 1.3 & 0.13 & 2.9 & 0.28 \\
\hline$C R$ & 1.1 & 0.69 & 9.5 & 0.90 & 5.0 & 0.07 & 1.7 & 0.16 & 3.8 & 0.37 & 2.0 & 0.41 & 4.6 & 0.45 & 4.8 & 0.44 \\
\hline CRL & 1.1 & 0.69 & 1.5 & 0.13 & 3.2 & 0.30 & 2.5 & 0.22 & 1.8 & 0.16 & 3.0 & 0.22 & 1.5 & 0.15 & 2.9 & 0.44 \\
\hline CRV & 1.1 & 0.69 & 2.7 & 0.24 & 10.0 & 0.95 & 4.1 & 0.41 & 3.9 & 0.37 & 4.1 & 0.46 & 3.7 & 0.35 & 5.1 & 0.50 \\
\hline CRVL & 1.1 & 0.69 & 2.6 & 0.24 & 2.5 & 0.23 & 2.5 & 0.23 & 2.5 & 0.23 & 2.9 & 0.25 & 2.5 & 0.24 & 3.2 & 0.30 \\
\hline$x$ & 1.1 & 0.69 & 4.1 & 0.38 & 4.6 & 0.44 & 2.3 & 0.22 & 2.8 & 0.25 & 2.8 & 0.31 & 2.7 & 0.27 & & \\
\hline
\end{tabular}




\section{DINAMICA DE NUTRIENTES Y MATERIA ORGANICA DEL SUELO}

Contenido de nutrientes

La aplicación de cenizas en la superficie del suelo resultó en un incremento significativo de $\mathrm{pH}$ y concentración de nitrógeno y decrecimiento en la acidez y el porcentaje de saturación de aluminio (Cuadro 6).

\section{Cuadro 6}

Características químicas del suelo en el bosque secundario original y después de la Sexta cosecha.

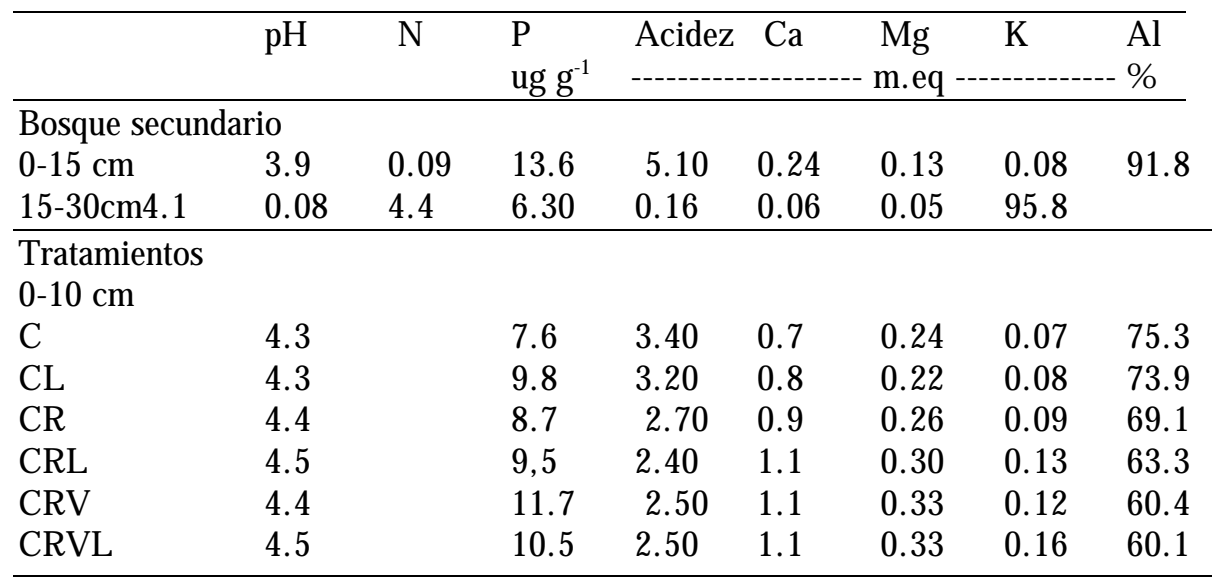

El pH y el contenido de nutrientes del suelo varió significativamente con el tiempo: No hay efecto de la actividad de las lombrices ni los tratamientos orgánicos. $\mathrm{El}$ pH se ha incrementado constantemente por encima de 4.7 en la segunda cosecha después de la incorporación de cenizas dentro del suelo. Cuando el $\mathrm{pH}$ decrece la actividad de las lombrices no tiene efecto sobre este parámetro. El porcentaje de saturación de aluminio ha decrecido hasta valores mínimos de $35 \%$ y ha fluctuado entre 60 y $75 \%$ (Figura 15).

El M g, Ca, P y K han decrecido regularmente durante los 6 períodos de cosecha. Esta disminución de $\mathrm{Ca}, \mathrm{Mg}$ no alcanzó los niveles del contenido de estos elementos en el bosque secundario mostrándose ligeramente superiores.

En cambio los niveles de $\mathrm{K}$ y $\mathrm{P}$ estuvieron por debajo de los niveles del bosque secundario (Figura 16) 

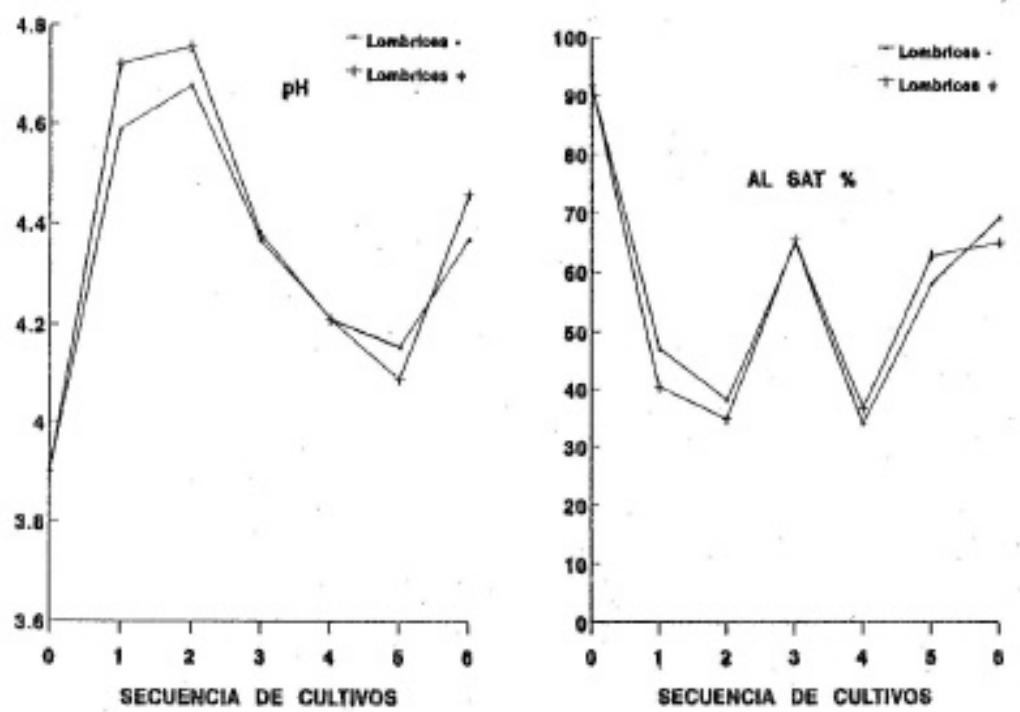

Figura 15. V ariaciones en pH e índice de saturación de aluminio.

\section{Cambio en los contenidos de $\mathrm{C}$ y $\mathrm{N}$}

Después de la primera cosecha, el contenido de $\mathrm{C}$ decrece constantemente en todos el tratamientos. No hay un efecto significativo de la calidad y cantidad de insumo orgánico.

Después de la quinta cosecha, el patrón de cambio y el contenido de $\mathrm{C}$ fue significativamente más bajo en el tratamiento con lombrices que sin lombrices. En la sexta cosecha se mantiene la tendencia de decrecimiento, pero mucho menos que en la quinta cosecha con sólo un 8. 1 \% (Figura 17), no existiendo diferencias. 

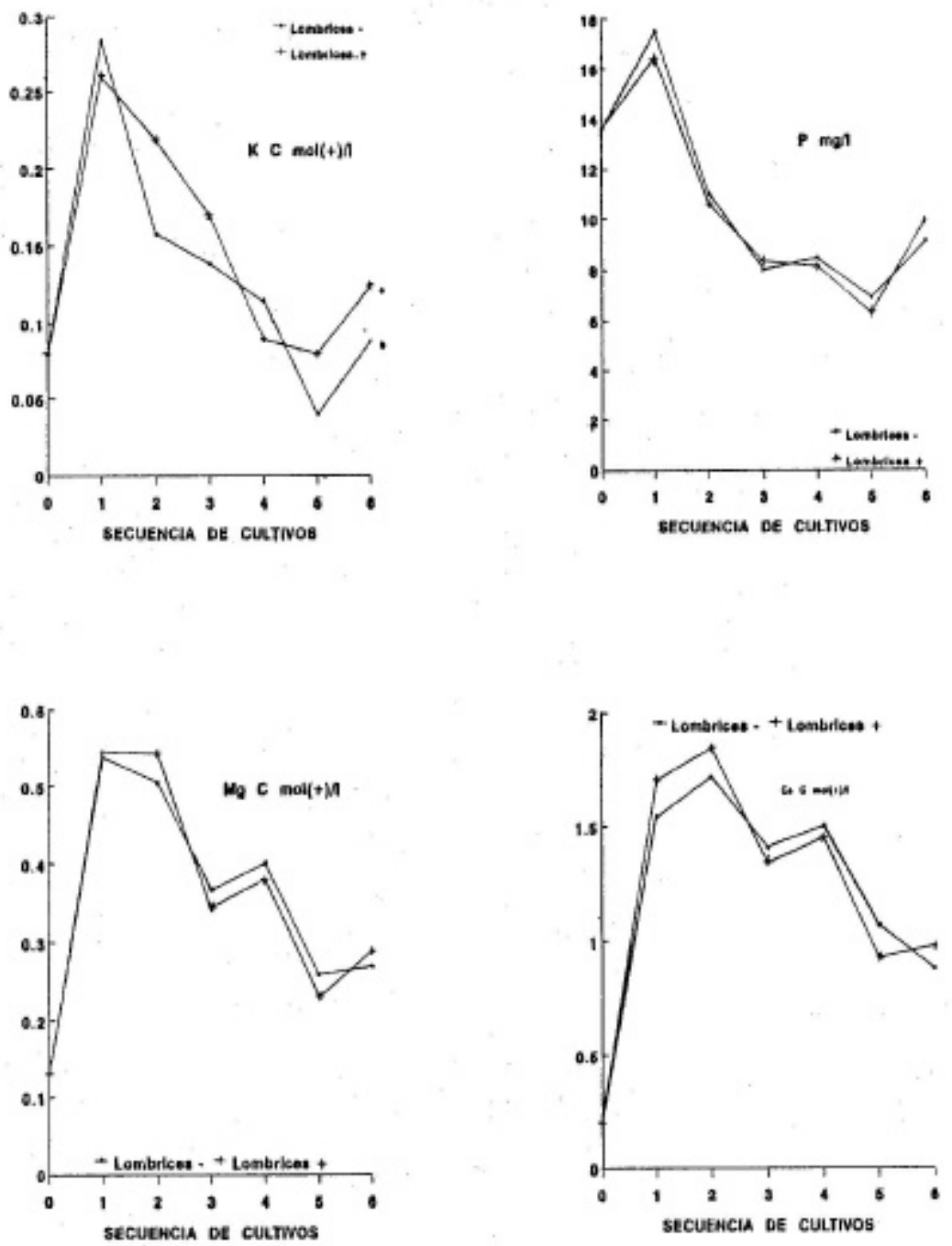

Figura 16. V ariaciones del contenido de nutrientes en el suelo. 


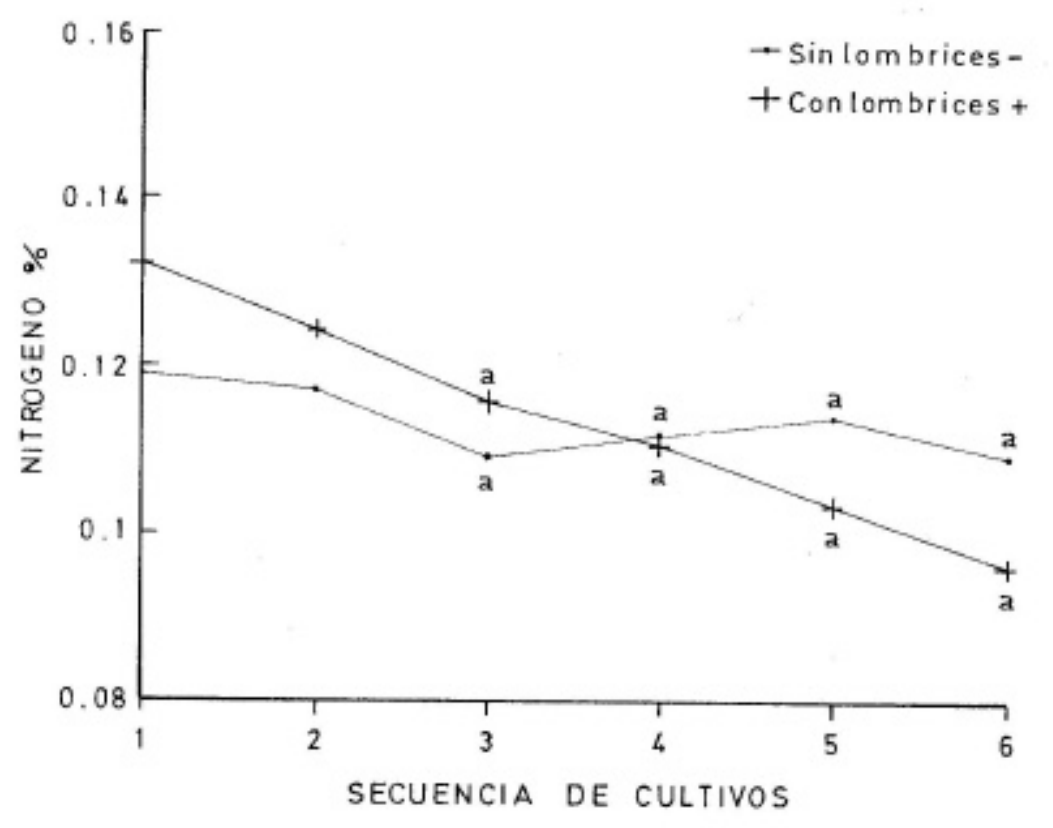

Figura 17. Variaciones del contenido de $\mathrm{C}$ a la profundidad de $0-10 \mathrm{~cm}$ con el tiempo y tratamientos. 
El contenido de $\mathrm{N}$ fue mayor en el tratamiento con lombrices durante las tres primeras cosechas; el promedio de diferencia durante este período fue (18\%) pero no fue significativa. En la quinta cosecha, la tendencia fue lo opuesto, pero no fue significativa la diferencia; manteniéndose el decrecimiento hasta la sexta cosecha, con una diferencia de $12 \%$ menos para los tratamientos con lombriz (Figura 18).

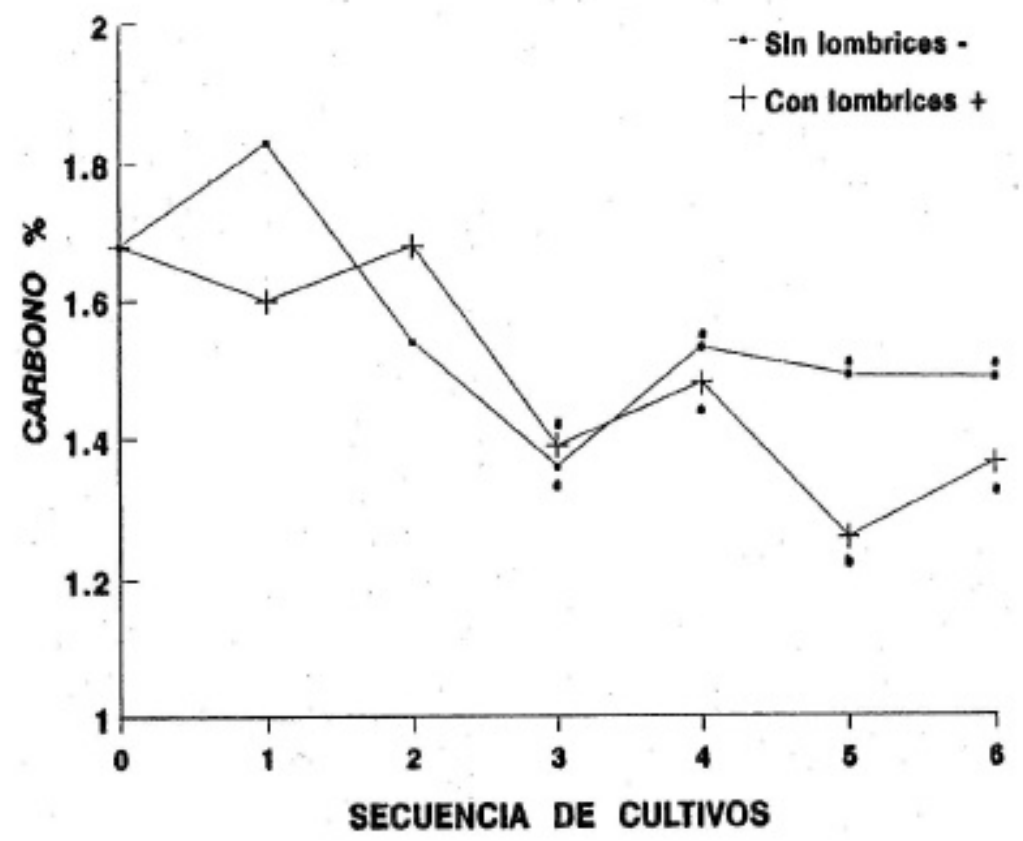

Figura 18. Variaciones del contenido de $\mathrm{N}$ a la profundidad de $0-10 \mathrm{~cm}$ con el tiempo y tratamientos. 


\section{ACTIVIDAD METABOLICA EN EL SUELO}

\section{Biomasa microbiana}

Las fluctuaciones del $\mathrm{N}$-microbial en el suelo indicará la conversión del $\mathrm{N}$-mineral dentro de formas orgánicas, inaprovechables por muchas plantas. Otra vez, una disminución general en todos los estados sucesivos de cultivo es consistente con el progresivo declive observados en los nitratos acumulados en el suelo. Un efecto remanente depresivo en ambas profundidades, en restos de raíz de maíz, menos marcado en caupí, no parece ser influenciado por la presencia 0 ausencia de lombrices.

En la primera cosecha no hubo diferencia significativa entre tratamientos en biomasa microbiana a la profundidad de $0-10 \mathrm{~cm}$. La presencia de lombrices no tuvo efecto significativo. En el segundo cultivo (arroz), hubo diferencia significativa entre tratamientos en biomasa microbiana. $L$ a presencia de lombrices no tuvo efecto significativo. En el cultivo de caupí no hubo diferencia significativa entre tratamientos, tampoco hubo efecto de la presencia de lombrices en las dos profundidades. En la cuarta rotación no hubo diferencia significativa entre tratamientos a la profundidad de $0-10 \mathrm{~cm}$. En ambas profundidades no hubo efecto de la inoculación de lombrices. En la sexta cosecha, la biomasa microbiana es mayor en los tratamientos sin lombriz. (Figura 19).

Acumulación de nitratos

Es interesante remarcar que en suelos Ultisoles, las numerosas poblaciones activas de nutrientes no están produciendo una acumulación de amonio.

Al final de cada cosecha, el suelo fue muestreado en estratos de 0-10 y 10-20 cm ambos para $\mathrm{N}$-mineral y biomasa microbiana.

En la capa de 0-10 cm, se observó una declinación en $\mathrm{NO}_{3}$ aprovechable por las plantas en el transcurso de la rotación de los cultivos. El efecto residual de caupí, sin embargo, se ha mantenido estable.

Al comienzo, las lombrices mostraron un efecto positivo y una reducción marcada al final. La presencia de rastrojo no mostró influencia. El abono verde parece que primero mejora la acumulación de nitrato y después provoca su agotamiento. 


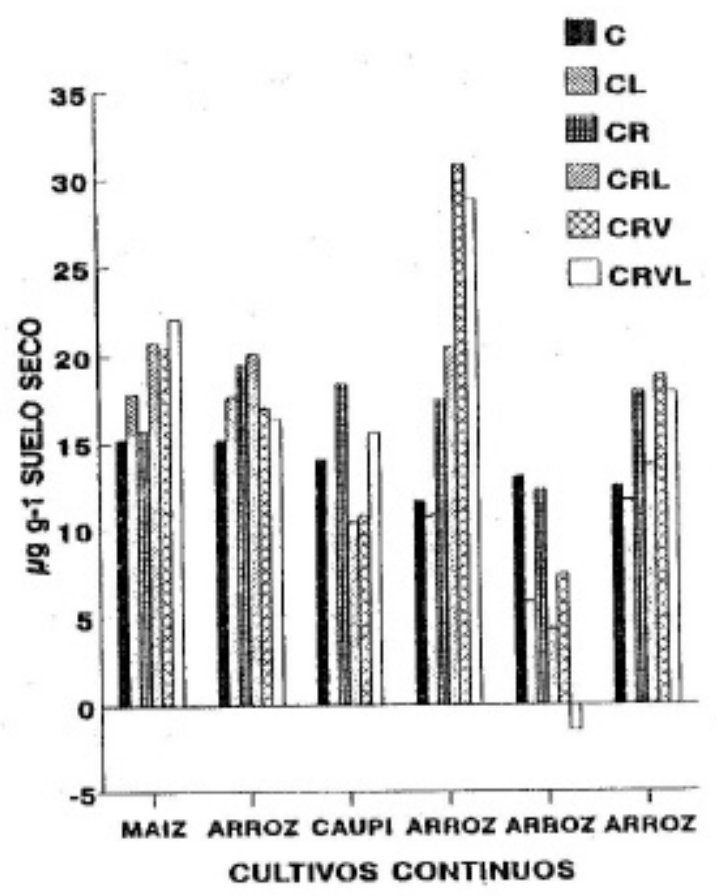

Figura 19. Cambios de $\mathrm{N}$-Biomasa microbiana durante los 6 periodos sucesivos de cosecha la profundidad de $0-10 \mathrm{~cm}$.

A la profundidad de $10-20 \mathrm{~cm}$, en la velocidad del $\mathrm{N}$-mineral y biomasa microbiana con el tiempo fue similar al de la capa superficial, pero con valores más bajos. El efecto residual de raíces de caupí se muestra completamente disminuido. Para las plantas en las parcela con lombrices. Un completo agotamiento de $\mathrm{NO}_{3}-\mathrm{N}$ tienen lugar después de 6 cosechas en todos los casos (figura 20). 


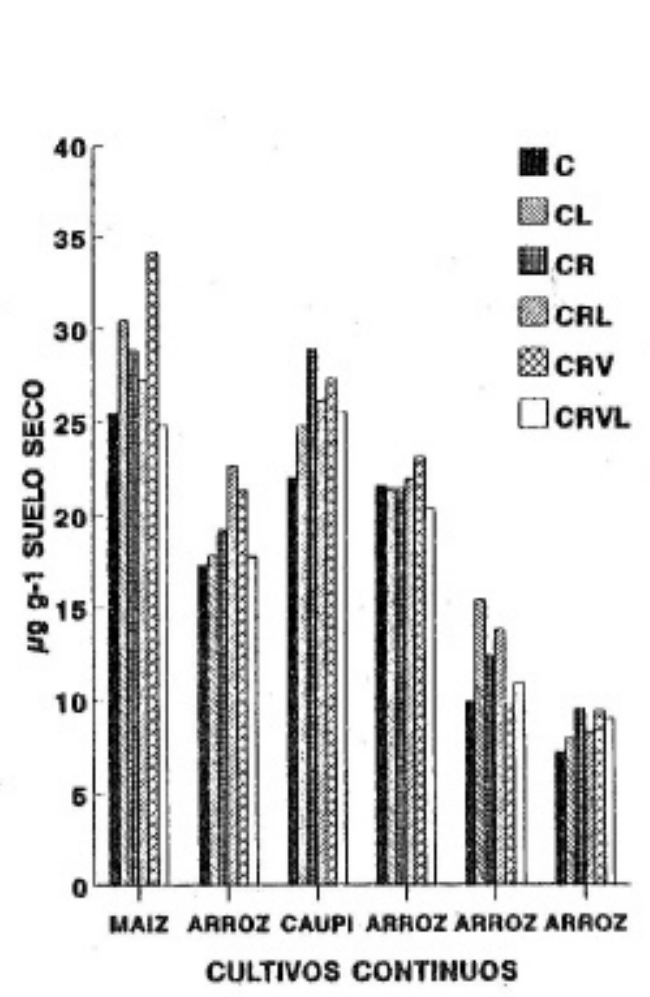

Figura 20. Cambios del potencial de nitrificación durante los 6 períodos sucesivos de cosecha.

\section{DISCUSION}

Dependiendo del tipo de manejo que el hombre le da al suelo se verán afectados las comunidades de fauna que habitan en forma natural el suelo. Los cultivos perennes presentan una menor diversidad de comunidades que el ecosistema original. Los cultivos anuales generalmente presentan una fauna casi agotada con bajas densidades de lombrices, con excepción de los termites que no son 
afectados. En parcelas experimentales se ha demostrado que la actividad de las lombrices puede resultar en un incremento significativo en la producción en pequeña escala. También se dan cambios en los nutrientes y en las propiedades físicas del suelo.

Los rendimientos de granos en el primer cultivo de maíz fluctuaron de 1. 1 a $2.1 \mathrm{~T} \mathrm{ha}^{-1}$. El mayor rendimiento se obtuvo en el tratamiento con rastrojo + abono verde leguminosa, que es una tonelada menos a los rendimientos obtenidos con tecnología de altos insumos (utilización de fertilizantes, pesticidas y maquinaria) en los campos experimentales de la estación. Los rendimientos a nivel local oscilan entre 1.2 a $1.5 \mathrm{~T} \mathrm{ha}^{-1}$ con sistema tradicional de uso del suelo que es similar al tratamiento control (suelo desnudo). El mayor rendimiento con rastrojo y abono verde más lombrices se debe a la incorporación adicional de nutrientes en los insumos orgánicos y su rápida descomposición y los nutrientes liberados fueron aprovechados por las plantas y conservadas en muchos casos en los turrículos de las lombrices (Cuadro 2).

En la segunda cosecha la producción de arroz fue de $0.77 \mathrm{~T} \mathrm{ha}^{-1}$ para el tratamiento control (suelo desnudo) y $1.49 \mathrm{~T}^{-1}$ para el tratamiento con rastrojo en la superficie. Estos rendimientos están en el rango de producción local en terrenos de altura. Los tratamientos que tuvieron lombrices incrementaron en un $88 \%$ más en comparación a los tratamientos sin lombrices, este incremento se debe a la presencia de agua en el suelo acumulado en los turrículos de las lombrices y que las plantas lo aprovecharon en una época determinada de su crecimiento y llenado de granos. Similar tendencia se observa en la producción de rastrojo y raíces.

En la cosecha de caupí no tuvo efecto la presencia de lombrices en la producción de granos, al contrario hay una decrecimiento de $-5 \%$ con relación a los tratamientos sin lombrices, aumentando el decrecimiento para rastrojo y raíces (Cuadro 2). Este cultivo tiene un requerimiento mínimo de agua en el suelo durante su ciclo vegetativo; pasa lo contrario con actividades y en caso contrario optan por el enclosamiento hasta que las condiciones se mejoren. Aun en estas condiciones los rendimientos obtenidos en los tratamientos con rastrojo + abono verde son similares a los rendimientos locales con promedios de $0.8 \mathrm{~T}^{\mathrm{h}} \mathrm{h}^{-1}$ (Guillén, 1991).

En la cuarta cosecha se obtuvo rendimientos en un rango de 0.73 a $2.00 \mathrm{~T}$ ha $^{-1}$. La presencia de lombrices produjo un incremento del $40 \%$ en los rendimientos de granos. $L$ a reducción de los rendimientos en el segundo cultivo se debieron a los períodos secos en que se produjo el déficit de agua. Esta falta de agua en el suelo dificulta la actividad de las lombrices, y el reciclaje de nutrientes incorporado en los insumos orgánicos ya que presentaron una tasa de descomposición más lenta. - 
En la quinta rotación con arroz los rendimientos oscilan entre 0.86 a $1.61 \mathrm{~T}$ ha-1. Hubo un efecto negativo con la presencia de lombrices, con un decrecimiento de $-43 \%$ en la producción de granos. Similar tendencia fue observada en rastrojo y raíces. Este decrecimiento se debe principalmente al déficit de agua en el suelo en las épocas críticas del cultivo (inicio de floración hasta el llenado de granos). A demás las lombrices tienen un requerimiento mínimo de agua en el suelo para su crecimiento, reproducción, y actividad. Todo esto ha producido una fuerte competencia por agua con las plantas, también se presenta acelerado agotamiento de la fertilidad del suelo en presencia de lombrices. En un inicio la actividad de las lombrices mejora la disponibilidad de los nutrientes siendo aprovechados por las plantas, pero después estos nutrientes se agotan y su efecto es adverso. La producción fue más alta en los tratamientos con rastrojo y rastrojo + abono verde (Cuadro 2).En la sexta cosecha se obtiene un incremento del $51 \%$ con presencia de lombrices; este incremento se debe probablemente a la abundante Iluvia caída en la zona en las épocas críticas de requerimiento de agua del arroz.

En la Figura 12 se presentan los cambios en la densidad aparente durante la producción de 6 cultivos consecutivos con y sin inoculación de lombrices de tierra. Como se observa en la Figura 12, se dan cambios en la densidad aparente cultivo tras cultivo y estos cambios siguen una tendencia variable de subida y bajada que se puede deber a las diferentes tasas de descomposición de los residuos, niveles de humedad del suelo en época seca y húmeda. La densidad aparente para el tratamiento con lombrices sigue una tendencia más uniforme de subida cultivo tras cultivo.El efecto mismo de las lombrices en descomponer el residuo o los abonos verdes uniformiza estos cambios en la densidad aparente y tiende a ser menos variables campaña tras campaña. Lo que sí está claro es que las lombrices aumentan la densidad aparente debido a la remoción y mezclado de partículas y algunas veces sellado o impermeabilización de la superficie del suelo por efecto de los turrículos.

En general, los valores máximos de densidad parente obtenidos son de $1.22 \mathrm{gr} / \mathrm{cm}^{3}$ que son ideales para un sistema de manejo continuo de cultivos con bajos insumos. En estos suelos de muy alta percolación este aumento en la densidad aparente es muy beneficioso para mantener la disponibilidad de agua, evitar la pérdida de nutrientes por lixiviación y evitar el estrés de agua en las épocas secas.

Las lombrices incrementaron la densidad aparente del suelo de valores iniciales de 1.10 a $1.18 \mathrm{~g} \mathrm{~cm}^{-3}$ en los tratamientos con lombriz y un decrecimiento en los tratamientos sin lombriz de 1.10 a $1.09 \mathrm{~g} \mathrm{~cm}^{-3}$. LoS incrementos oscilan en un rango de 7.0 a $10.0 \%$ con relación a los tratamientos sin lombrices durante los 6 períodos de cosecha. Estos resultados son similares a los encontrados por Lal(1989) con un incremento significativo en la densidad aparente con la duración del tiempo de cultivo en las capas de 0-5 cm y $5-10 \mathrm{~cm}$ 
respectivamente con valores iniciales de 1.02 - 1.16 a 1.43 y $1.65 \mathrm{~g} \mathrm{~cm}^{-3}$ hasta concluir el ciclo de cultivo.

\section{CONCLUSIONES}

1) La inoculación de lombrices tuvo efectos variables sobre las propiedades del suelo y la producción de las plantas. A unque el agotamiento de materia orgánica y las reservas de nutrientes no fue impedido, la estructura física del suelo y la actividad metabólica de la microflora fue modificado.

2) El sistema de cultivo de bajos insumos que asocia en su incorporación rastrojo de cosecha y abono verde de leguminosas con mulch en la superficie y la inoculación de pontoscolex corethrurus puede mantener mayor producción que el sistema tradicional por largos períodos.

3) La dinámica de los nutrientes del suelo con lombrices fue similar al tratamiento sin lombrices. Hubo un incremento inicial durante la primera cosecha (debido a la adición de nutrientes en la ceniza).

4) L L densidad aparente se incrementó y la infiltración inicial decreció en el tratamiento con lombrices comparado al tratamiento sin lombrices debido a la mezcla del suelo.

\section{BIBLIOGRAFIA}

BAROIS, 1; VERDIER, B,; KAISER, P; \& LAVELLE, P 1987. Role of the tropical earthworms Pontoscolex corethrurus in the nitrogen cycle and particulary in the anaerobic nitrogen fixation. In: On Earthworms ( $A$. Bonvicini Pagliari et P. Omodeo (eds). M ucchi editore, Selected Symposia M onographs, 2:281-296.

BLANCHART, E, LAVELLE, P \& SPAIN, A.V. 1990. Effects of biomass and size of Millsonia anomalia (Oligochaeta, A canthrodrilidae) on particle agregation in a tropical soil in the presence of Panicum maximum. Biology Fertility Soil, 9: in press.

COCHRAME, 1. AND P.A. SÁNCHEZ. 1982. Land resources, soils properties and their management in the amazon region; $A$ state of knowledge report. p. 138-209. In S.B. Hecht (cd) A mazon land use research. CIAT, Cali, Colombia. 
DANGERFIELD， M. 1989. A bundance, biomass and diversity of soil macrofauna in savanna woodland and associated managed habitats. Pedobiologia 34.

ESTRIBI CHAVARRIA, C.A. 1978.Cambios edáficos e hidrológicos derivados de la conversión de bosques a pasto y charral (pasto abandonado) en una zona montañosa húmeda de Costa Rica. Tesis Mag. Sc. Turrialba, Costa Rica. CATIE 139 p.

GUILLEN, W. 1991. Memorias del Informe anual. Programa de Investigación de Leguminosas de Grano. Estación Experimental "San Ramón" de Y urimaguas.

$L A L, R$. 1974. No-tillage effects on soil properties and maize production in W estern Nigeria. Plant and Soil, 40: 321-33 1.

LAVELLE, P. 1987. Biological processes and productivity of soils in the humid tropics. In: J. Lovelock and Dickinson (eds). Geophysical of A mazonia. John Wiley, N ew Y ork. pp 175-213.

LAVELLE, P. ami PASHANASI, B. 1989. Soil macrofauna and land management in Peruvian A mazonia (Y urimaguas, Loreto). Pedobiología 33: $283-291$.

LAVELLE, P; BLANCHART, E.; MARTÍN, A.; MARTÍN, 5,; SPAIN, A.V.,; TOUNTALN, F.; BAROIS, 1. AND SCHAEFER, R. 1991. The impact of soil fauna on the properties of soils in the humid tropics. Soil Science Society of A merica J ournal, in press.

PASHANASI, B.; MELENDEZ, 0.; SZOTT, L. AND LAVELLE, P. 1992. Effect of inoculation with the endogenic earthworm Pontoscolex corethrurus (Glossoscolecidae) on $\mathrm{N}$ availability soil microbial biomass and the growth of tree tropical fruit seedlings in a pot experimental. Soil Biology \& Biochemistry, Vol. 24 No 12. pp. 1655-1659.

SWIFT, M.J. 1987. Tropical Soil Biology and Fertility (TSBF). Planning for Research, Biol. mt. 9, 24p. 